\title{
Combined High-speed and High-lift Wing Aerodynamic Optimization Using a Coupled VLM-2.5D RANS Approach
}

\author{
M. Parenteau ${ }^{\mathrm{a}, *}$, É. Laurendeau ${ }^{\mathrm{a}, * *}$, G. Carrier ${ }^{\mathrm{b}}$ \\ ${ }^{a}$ Department of Mechanical Engineering, École Polytechnique de Montréal, Montréal, Québec, H3C 3A7, \\ Canada \\ ${ }^{b}$ DAAA, Department of Aerodynamics, Aeroelasticity, Acoustics, ONERA, The French Aerospace Lab, \\ F-92190, Meudon, France
}

\begin{abstract}
The paper presents a numerical framework for the aerodynamic analysis of aircraft wings in transonic cruise and take-off/landing compatible with preliminary and conceptual design phase requirements based on the Non-Linear Vortex Lattice Method (NL-VLM). The purpose of this work is to demonstrate the applicability of the VLM-2.5D RANS approach for aircraft design optimization. The algorithm captures wing sweep effects, important in the transonic regime and near $C_{L_{\max }}$ conditions, by a stripwise viscous-inviscid coupling strategy with an infinite-swept wing (2.5D) Reynolds-Averaged Navier-Stokes (RANS) solver. Aerodynamic forces are evaluated through spanwise integration of the 2.5D RANS solutions and a trefftz-plane analysis of the VLM solver. The framework allows calculations of single and multi-element configurations without modifying the VLM mesh. A novel $C_{L_{\max }}$ criteria is proposed based on recently observed stall-cells patterns that captures $C_{L_{\max }}, \alpha_{\max }$ and the spanwise location of the stall, which represent important design parameters. The applicability of the framework to aircraft design is demonstrated by embedding the analysis tools into a gradient-free Covariance Matrix Adaptation Evolution Strategy. After a verification phase, validation is performed on high-speed, high-lift and combined high-speed/high-lift optimisations cases. In particular, the capability of the numerical algorithms towards multi-topology optimisation is demonstrated.
\end{abstract}

\section{Introduction}

Aircraft design is made of three distinct phases, preliminary, conceptual and detailed. These phases aim at different objectives so that different tools are necessary. In conceptual design, the aerodynamic analysis comes mainly from knowledge-based and low-fidelity methods with low computation cost, because hundreds if not thousands of concepts are

\footnotetext{
${ }^{*}$ Corresponding author

**Principal corresponding author

Email addresses: matthieu.parenteau@polymtl.ca (M. Parenteau ), eric.laurendeau@polymtl.ca (É. Laurendeau ), gerald.carrier@onera.fr (G. Carrier) 


\section{Nomenclature}

\begin{tabular}{|c|c|c|c|}
\hline $\begin{array}{l}\alpha, \text { AoA } \\
\alpha_{\max }\end{array}$ & $\begin{array}{l}\text { Angle of attack } \\
\text { Angle of attack at } C_{L_{\max }}\end{array}$ & $C l_{i n v}$ & $\begin{array}{l}\text { Inviscid sectional lift coeffi- } \\
\text { cient }\end{array}$ \\
\hline$\Delta \alpha$ & Viscous corrections & $C l_{v i s c}$ & Viscous sectional lift coeffi- \\
\hline$\Gamma$ & Circulation magnitude & & it \\
\hline$\lambda$ & Aspect ratio & $d l$ & $\begin{array}{l}\text { Incremental vortex segment } \\
\text { of length }\end{array}$ \\
\hline$\phi, \Lambda$ & Sweep angle & $L E$ & Leading Edge \\
\hline$A$ & Geometry influence matrix & $S$ & Wing reference area \\
\hline$b$ & Wing span & N & wing rererence area \\
\hline$C_{D}$ & 3D drag coefficient & $t$ & Wing thickness \\
\hline$c_{k}$ & Kink chord & $T E$ & Trailing Edge \\
\hline$C_{L}$ & 3D lift coefficient & $W_{t o}$ & Aircraft takeoff weight \\
\hline$C_{M}$ & 3D pitching moment coeffi- & $W_{w}$ & Weight of the wing \\
\hline & cient & $W_{z f}$ & Aircraft zero fuel weight \\
\hline$c_{r}$ & Root chord & $x_{c p}$ & Pressure center position \\
\hline$C_{L_{\max }}$ & Maximum lift coefficient & $x_{r e f}$ & Reference point for pitching \\
\hline$C l_{\alpha}$ & Lift curve slope & & moment calculation \\
\hline
\end{tabular}

evaluated[1]. Moreover, nearly $80 \%$ of the life-cycle cost are induced by choices made in the conceptual phase[2]. The aerodynamic and structural analysis are particularly prone to errors due to the low-fidelity methods used and the complex interaction between the disciplines involved[2].

In a conceptual multi-disciplinary design optimization (CMDO) framework, the number of design variables remains low and the optimization is oriented towards the exploration of the design space[1]. A stochastic optimizer is often used at this stage with both singleobjective and multi-objective functions and requires thousand of function evaluations to converge toward an optimum. Fast turnaround aerodynamic tools are then necessary like inviscid potential methods. A linear potential method such as the Vortex Lattice Method (VLM) solves inviscid, attached and incompressible flows, which does not represent the flow physics over the aircraft. Therefore, non-linear effects like compressibility and flow separation cannot be captured by this method.

However, viscous correction can be applied to the VLM by using 2D RANS data or experimental data $[3,4,5,6,7,8]$. These methods are solved iteratively to find the viscous correction by changing the local angle of attack[3, 4, 9] or by using a local decambering approach[7]. These viscous correction methods have showed interesting accuracy[6, 10] compared to 
higher-fidelity solutions for low-speed $C_{L_{\max }}$ prediction[3] and transonic conditions[11]. One interesting feature presented by Gallay et al.[11] is the use of 2.5D RANS data to incorporate crossflow effects for a better $C_{L_{\max }}$ and shock wave prediction[6].

The goal of the current work is to perform aerodynamic optimization of wing planform using a VLM/2.5D RANS approach[6] with a Covariant Matrix Adaptation Evolution Strategy (CMA-ES)[12] optimizer. High-speed optimization is explored with low-speed multi-topology optimization as well. Moreover, a new spanwise stall detection criteria is introduced as an optimization constraint. Finally, low-speed and high-speed optimizations are combined together to explore the trade-off between high-speed and low-speed objective functions.

\section{Aerodynamic Solver}

\subsection{Vortex Lattice Method Extended to high Angle of Attack}

The wing is modeled as a thin surface represented by vortex rings (Figure 1). The bound

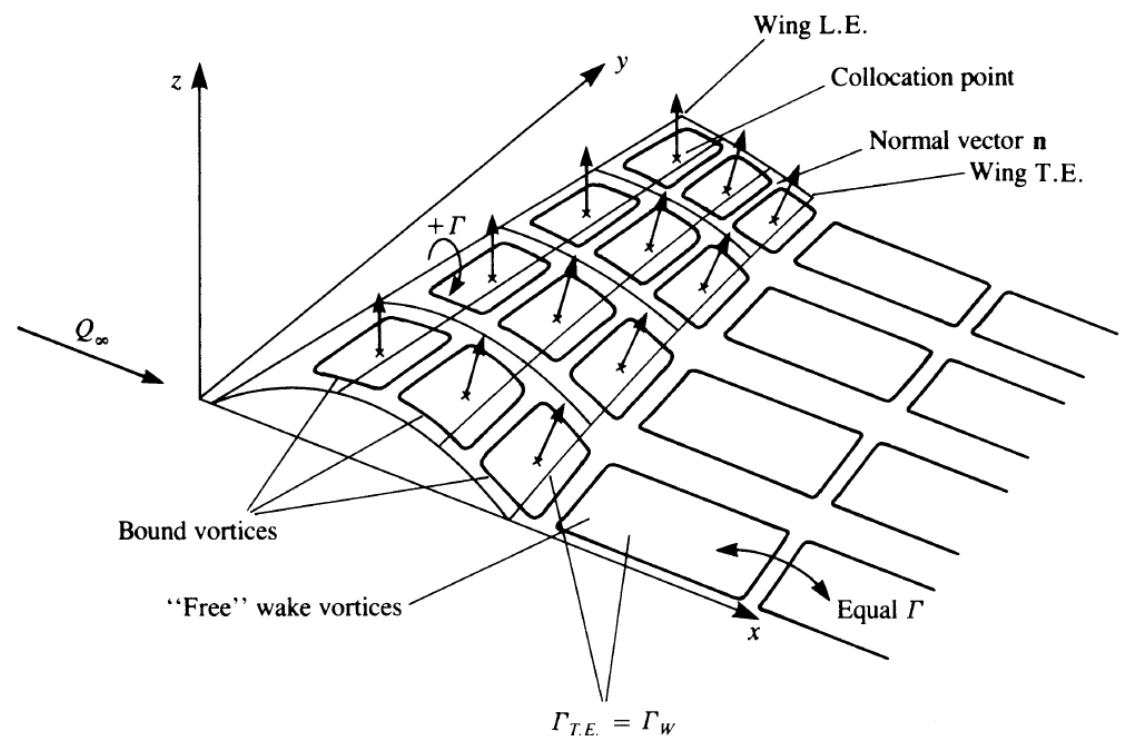

Figure 1: Representation of thin lifting surfaces (Source: [13]).

vortices are placed on the wing panel's $1 / 4$ chord and the Neumann boundary condition of no penetration is enforced at the collocation point located at the $3 / 4$ chord of the wing's panel. This is referred to as the 1/4-3/4 rule and it is a fundamental concept for Vortex Lattice Methods derived by Pistolesi[14]. By using a single panel vortex lattice in two-dimension (Figure 2), he found that by placing the vortex point at the $1 / 4$ chord and the boundary condition at the $3 / 4$ chord, the section lift curve slope corresponds exactly to the thin airfoil theory $(2 \pi)$. However, this is only true with the assumption of small angles of attack. The velocity induced $U$ at a distance $r$ from a vortex placed at c/4 with the boundary condition 
is represented by:

$$
U=U_{\infty} \sin (\alpha)=\frac{\Gamma}{2 \pi r}
$$

with Kutta-Joukowski formulation of lift and thin airfoil theory, but without assumption of small angles becomes:

$$
\begin{aligned}
\frac{1}{2} \rho U_{\infty}^{2} c 2 \pi \alpha & =\rho U_{\infty} \Gamma=\rho U_{\infty} 2 \pi r U_{\infty} \sin (\alpha) \\
\frac{1}{2} c 2 \pi \alpha & =2 \pi r \sin (\alpha) \\
r & =\frac{1}{2} \frac{\alpha}{\sin (\alpha)}
\end{aligned}
$$

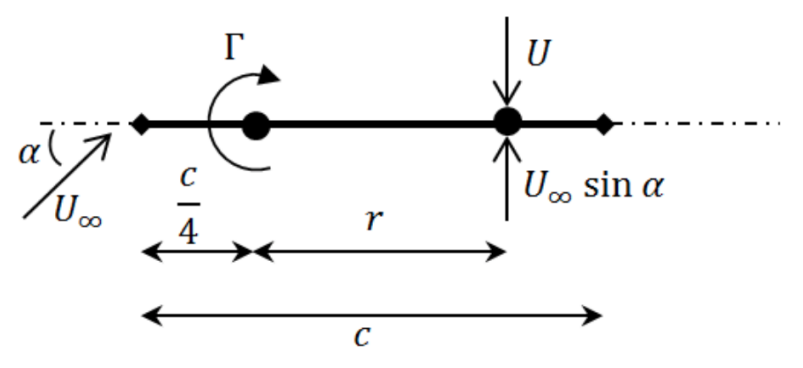

Figure 2: Control point and vortex location (Source: [6]).

Therefore, the collocation point must be corrected at high angles of attack to maintain a local lift curve slope of $2 \pi$. The influence of each vortex rings on each other is evaluated using the Biot-Savart Law and assembled into an influence matrix $A$. The boundary condition is incorporated in the right-hand side (RHS) and the linear system is solved for the unknown circulation $\Gamma$ (Equations 5).

$$
\begin{gathered}
\left(\begin{array}{cccc}
A_{1,1} & A_{1,2} & \cdots & A_{1, m} \\
A_{2,1} & A_{2,2} & \cdots & A_{2, m} \\
\vdots & \vdots & \ddots & \vdots \\
A_{m, 1} & A_{m, 2} & \cdots & A_{m, m}
\end{array}\right)\left(\begin{array}{c}
\Gamma_{1} \\
\Gamma_{2} \\
\vdots \\
\Gamma_{m}
\end{array}\right)=\left(\begin{array}{c}
R H S_{1} \\
R H S_{2} \\
\vdots \\
R H S_{m}
\end{array}\right) \\
R H S_{K}=(U, V, W)_{\infty} \cdot n_{K}
\end{gathered}
$$

The Vortex Lattice Method only models inviscid, incompressible and attached flows. Here, we include sectional data computed with a two-dimensional RANS flow solver to incorporate non-linear effects such as flow separation and shock waves. 


\subsection{5D RANS Flow Solver}

The viscous sectional data are generated with NSCODE[15], a two-dimensional RANS flow solver extended for infinite swept flows[16]. The 2D Navier-Stokes equations are solved with the addition of one scalar equation for the crossflow derived with infinite swept wing conditions in a rotated coordinate system normal to the sweep line, $\frac{\partial}{\partial y^{\prime}}=0$,

$$
\frac{\partial \rho v^{\prime}}{\partial t}+u^{\prime} \frac{\partial \rho v^{\prime}}{\partial x^{\prime}}+w^{\prime} \frac{\partial \rho v^{\prime}}{\partial z^{\prime}}=\frac{\partial \tau_{x^{\prime} y^{\prime}}}{\partial x^{\prime}}+\frac{\partial \tau_{y^{\prime} z^{\prime}}}{\partial z^{\prime}}
$$

where $v^{\prime}$ is the crossflow velocity in the sweep line direction. The $2.5 \mathrm{D}$ extension allows crossflow effects to be computed with a $2 \mathrm{D}$ mesh. There is no cells or halos required in the spanwise direction, thus reducing the computational cost and memory requirement compared to a similar infinite-swept wing 3D RANS flow solver calculation. The effect of incorporating crossflow with the infinite swept wing condition is demonstrated in Figure 3 where the same calculation is performed for different swept flows conditions in low-speed and high-speed regimes. The low-speed $C_{L_{\max }}$ is reduced with increasing sweep as well as the lift curve slope. In transonic conditions, the shock position is reduced from $50 \%$ without crossflow to $25 \%$ chordwise with a $30^{\circ}$ swept flow condition. The leading edge pressure peak is also increased with the sweep. These crossflow effects, when coupled to the VLM, allows a better maximum lift coefficient prediction for high-lift configurations and viscous drag estimation in transonic conditions. Since the chordwise pressure distribution is also better captured, the pitching moment estimation is also improved with 2.5D sectional data. Physical effects captured by the infinite swept wing condition are presented in Figure 4.

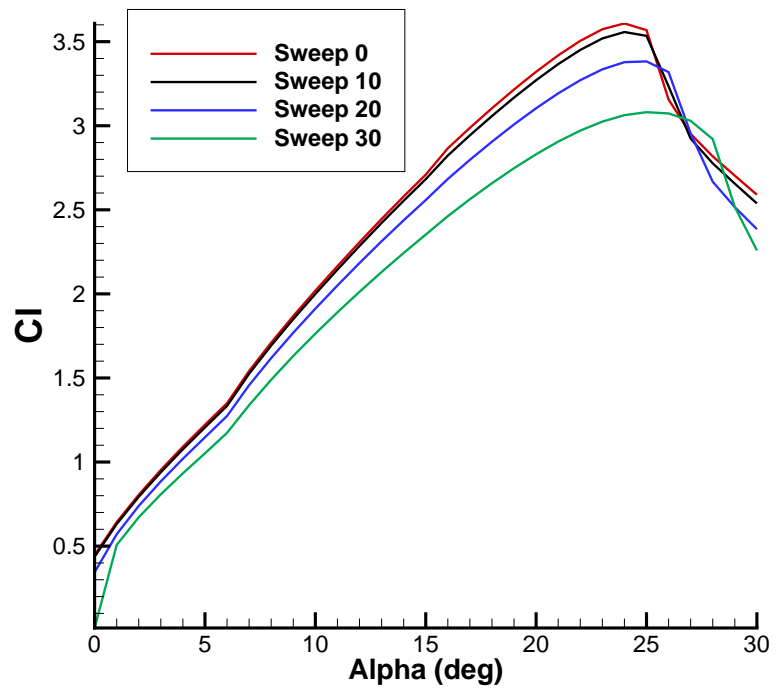

(a) Mach $=0.2$; Slat: $25^{\circ}$; Flap: $10^{\circ} ; \operatorname{Re}=1.3 E 6$.

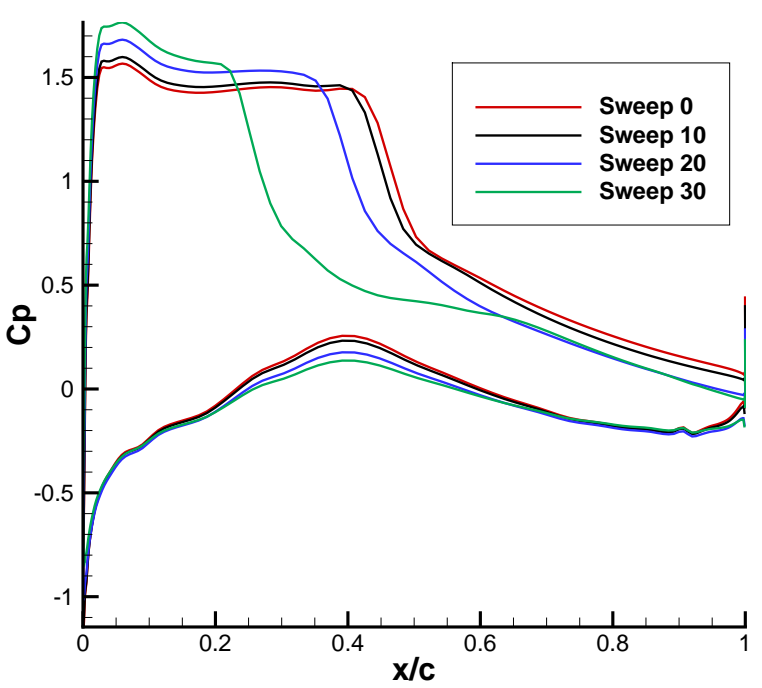

(b) Mach $=0.75 ;$ Alpha $=5^{\circ} ; R e=6.3 E 6$.

Figure 3: Lovell - Crossflow effects over the sectional aerodynamic characteristics. 


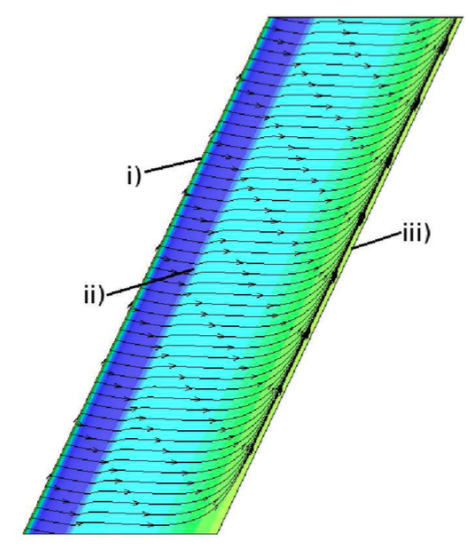

Figure 4: Physical effects captured by infinite swept wing RANS solution : i) stagnation region, ii) shock waves, iii) trailing-edge crossflow boundary-layer thickening/separation (Source: [11]).

\subsection{Viscous Coupling Algorithm}

Viscous corrections are incorporated using an alpha coupling algorithm[? 3, 6]. Viscous effects are introduced from sectional aerodynamic coefficients computed with a 2D/2.5D RANS flow solver. Corrections are applied iteratively on the local angle of attack, $\alpha_{2 D}$, in the right-hand side of the VLM system of equations until the inviscid sectional lift is equal to the viscous lift for each section spanwise. A typical implementation is presented by Algorithm 1. To better understand the coupling scheme, Figure 5 is used to demonstrate how the local effective angle of attack is calculated with 2.5D RANS data. For a local section

$$
C l_{\text {inv }}=C l_{\text {visc }}=2 \pi\left(\alpha-\alpha_{i}-\alpha_{0 l}-\Delta \alpha\right)
$$

where $\Delta \alpha$ is the viscous corrections, $\alpha_{i}$ the induced angle of attack and $\alpha_{0 l}$ the zero lift angle of attack. The effective angle of attack is defined as

$$
\alpha-\alpha_{i}=\alpha_{e}=\frac{C l_{i n v}}{C l_{\alpha}}+\alpha_{0 l}+\Delta \alpha
$$

with the viscous corrections defined as

$$
\Delta \alpha=\alpha_{3 D}-\alpha_{2 D} \cos \phi
$$

where $\phi$ is the sweep line used to generate the 2.5D RANS sectional data. The $\cos \phi$ term comes from the lifting-line potential equation for infinite swept wing conditions[17]. The $\cos \phi$ correction is necessary when using 2.5D RANS sectional data to ensure the correct local lift curve slope[18]. The local angle of attack is evaluated using a constant local lift curve slope of $2 \pi$. Therefore, the collocation point is calculated according to the local angle 
of attack as derived previously in Equation 4. Furthermore, since no camber is modeled the VLM model the zero list angle of attack, $\alpha_{0 l}$, is equal to zero. More details on the coupling algorithm can be found in [19].

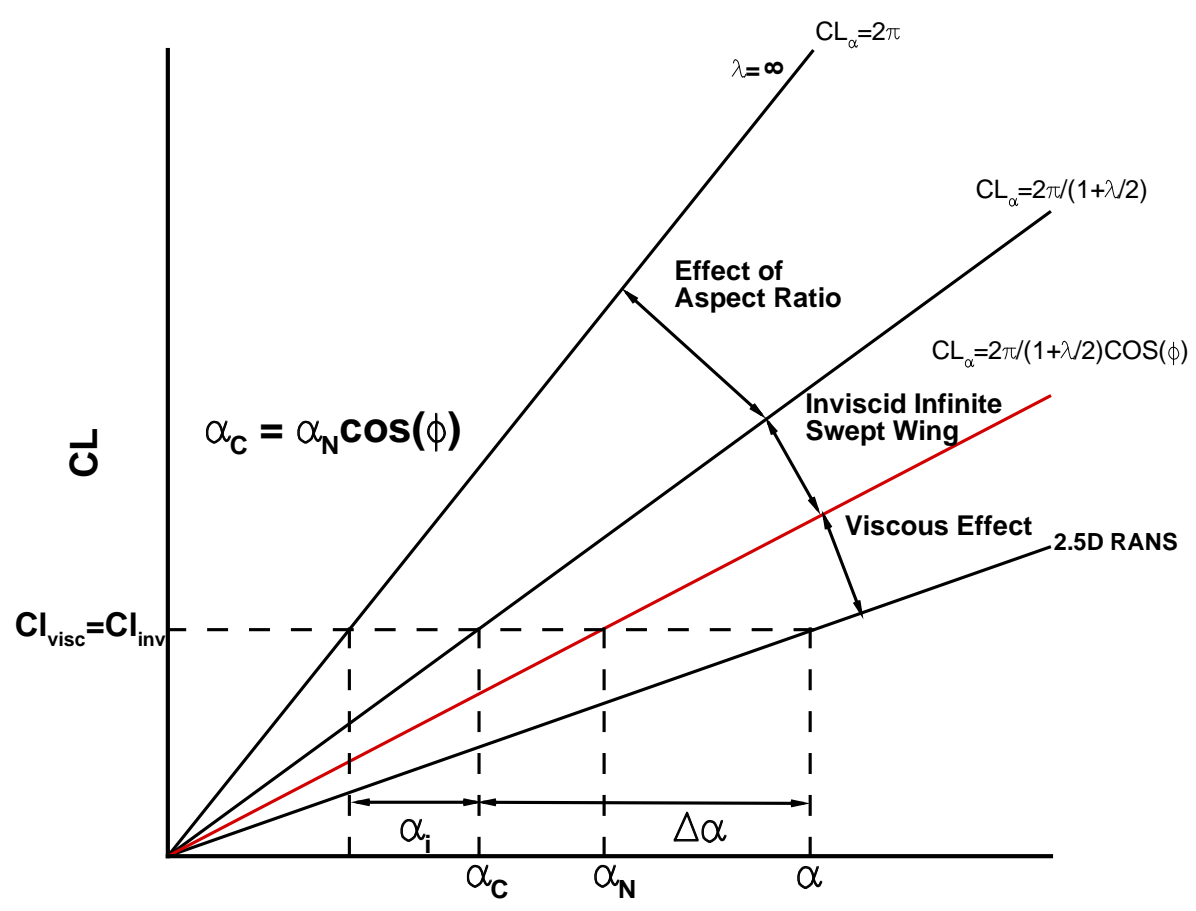

Figure 5: Viscous coupling algorithm.

One advantage of this approach is that it allows 3D multi-topology analysis without the need to generate complex 3D meshes required for 3D RANS solvers. Since complex flow physics is incorporated in the 2.5D RANS solution, the approach only requires $2 \mathrm{D}$ meshes for single and multi-element airfoil sections. These are easily amenable to automation, and in this work the overset approach is used to further simplify the multi-element analysis. Also, it was found in [6] that the VLM mesh can remain fixed and uncambered since the information is carried by the RANS solution (Figure 6). This also eases the VLM meshing which remains unchanged with regards to the different topologies defined along the span.

\subsection{Forces Calculation}

The total lift is evaluated by summing the incremental lift force of each panel with the Kutta-Joukowski theorem as follow:

$$
F=\sum_{i=1}^{N} \rho_{\infty} \Gamma_{i}\left(U_{\infty} \times d l\right)
$$




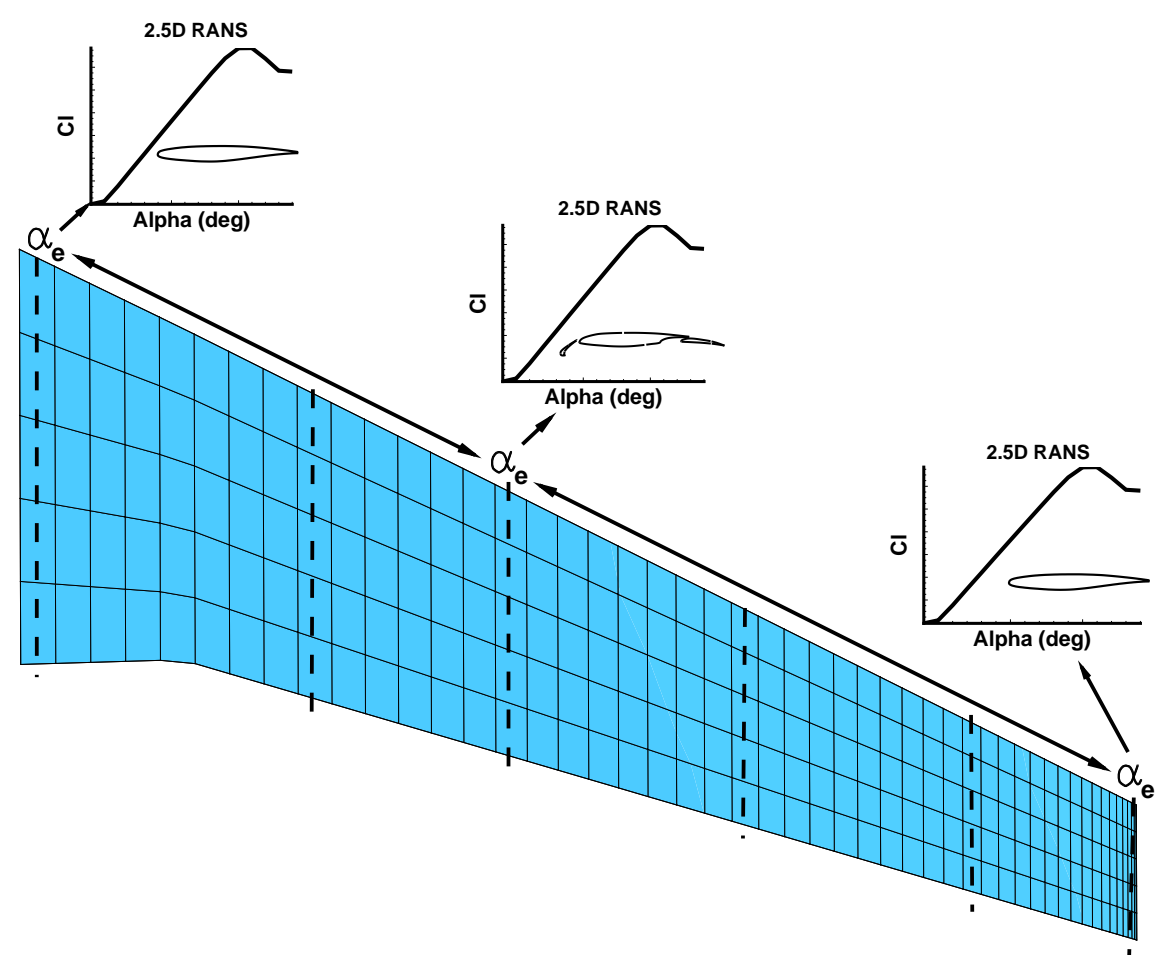

Figure 6: Viscous correction applied to the VLM.

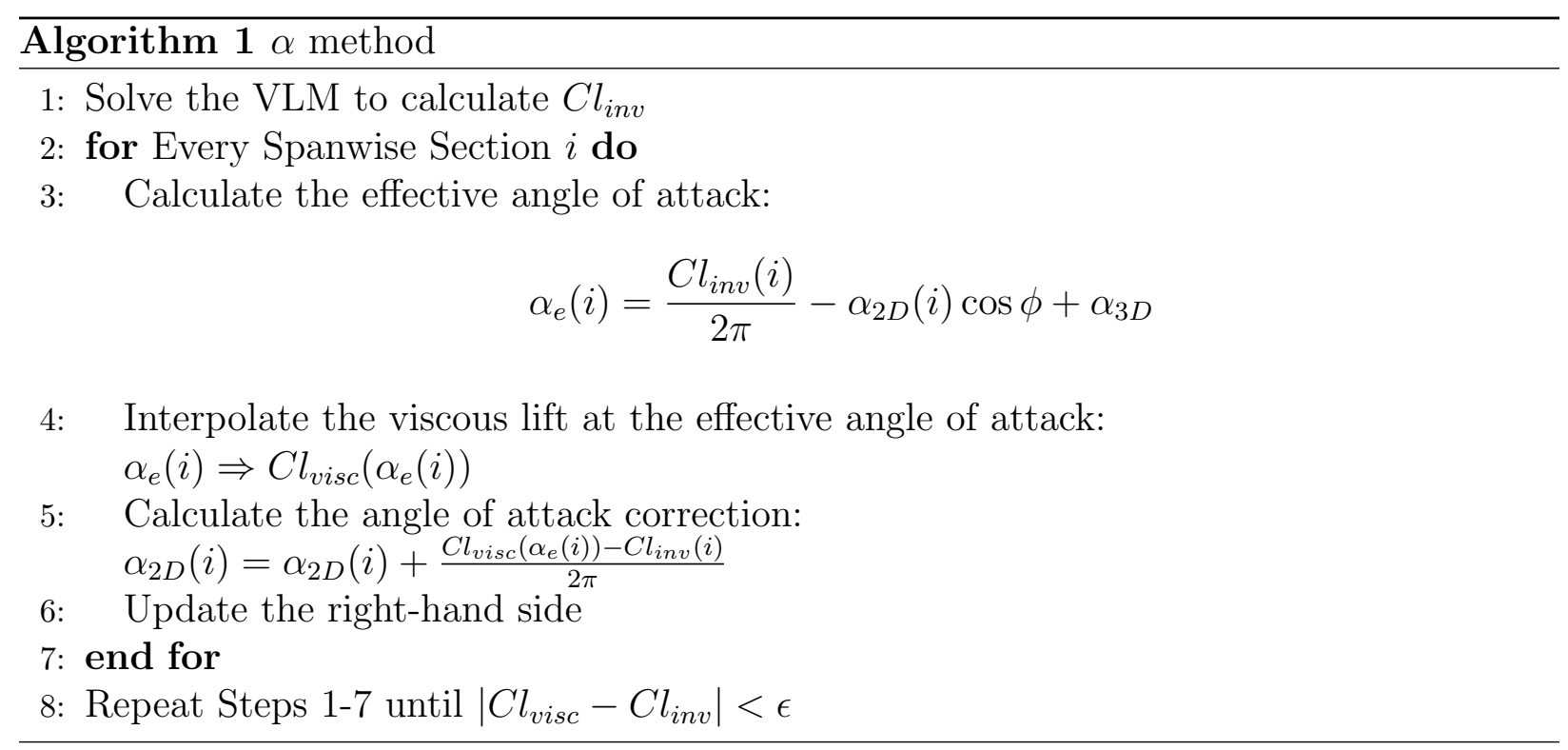

where $d l$ is the bound vortex filament. The lift force is calculated with the VLM while the drag estimation is divided into two contributions, induced drag and viscous drag.

$$
D_{\text {total }}=D_{\text {induced }}+D_{\text {viscous }}
$$


The induced drag is calculated by integrating the kinetic energy far behind the lifting body in a plane normal to the free stream called the Trefftz plane. For a lifting-line method, the surface integral can be transferred to a spanwise line integral over the wake trailing vortices as follows:

$$
D_{\text {induced }}=-\frac{\rho_{\infty}}{2} \int_{-b / 2}^{b / 2} \Gamma(y) w d y
$$

where $w$ is the induced velocity of the trailing vortices in the Trefftz plane. On the other hand, the viscous drag is calculated from the sectional RANS data and includes the friction and pressure drag. For each spanwise section, the viscous drag coefficient is interpolated from the local RANS data values at the corresponding effective angle of attack calculated by the coupling algorithm. A spanwise integration is then performed to obtain the total viscous drag:

$$
D_{\text {viscous }}=\frac{1}{2} \rho_{\infty} U_{\infty}^{2} \int_{-b / 2}^{b / 2} C_{D_{\text {viscous }}} c d y
$$

where $c$ is the local chord length. The 3D pitching moment is also evaluated by a spanwise integration of the local RANS pitching moment. The center of pressure for every spanwise section is evaluated using the local viscous lift and viscous drag interpolated at the corresponding effective AoA. The pitching moment relative to the $3 \mathrm{D}$ wing reference point is then computed and integrated spanwise:

$$
\begin{aligned}
x_{c p} & =\frac{\left(C_{m_{c / 4}}\right)_{v i s c}}{C_{l_{v i s c}}} \\
C_{m_{x_{r e f}}} & =\left[C_{l_{v i s c}} \cos \left(\alpha_{e}\right)+C_{d_{v i s c}} \sin \left(\alpha_{e}\right)\right] \frac{x_{r e f}+x_{c p}}{c} \\
M_{x_{r e f}} & =\frac{1}{2} \rho_{\infty} U_{\infty}^{2} \int_{-b / 2}^{b / 2} C_{m_{x_{r e f}}} c^{2} d y
\end{aligned}
$$

\subsection{Spanwise Stall Detection Scheme}

The VLM coupled with viscous sectional data is capable of capturing stall cells, numerically obtained by Spalart[20] using a spectral approach and Gallay[5] using the proposed approach. Stall cells are triggered when the lift-curve slope at a spanwise section becomes negative. In other words, when a streamwise section in the VLM reaches its viscous $C_{L_{\max }}$ computed with 2.5D RANS, a sudden change in lift appears spanwise forming a stall cell. Therefore, the appearance of the first stall cell is used as the criteria for the stall spanwise position. To do so, the spanloads before and after $\alpha_{\max }$ are compared and the highest $\Delta C l$ indicates the position of the stall cell and thus the spanwise stall position as demonstrated in Figure 7. This criteria essentially substitutes the lower fidelity method developed by Valarezo and Chin[21], and provides not only $C_{L_{m a x}}$, but also an accurate prediction of $\alpha_{\max }$. 


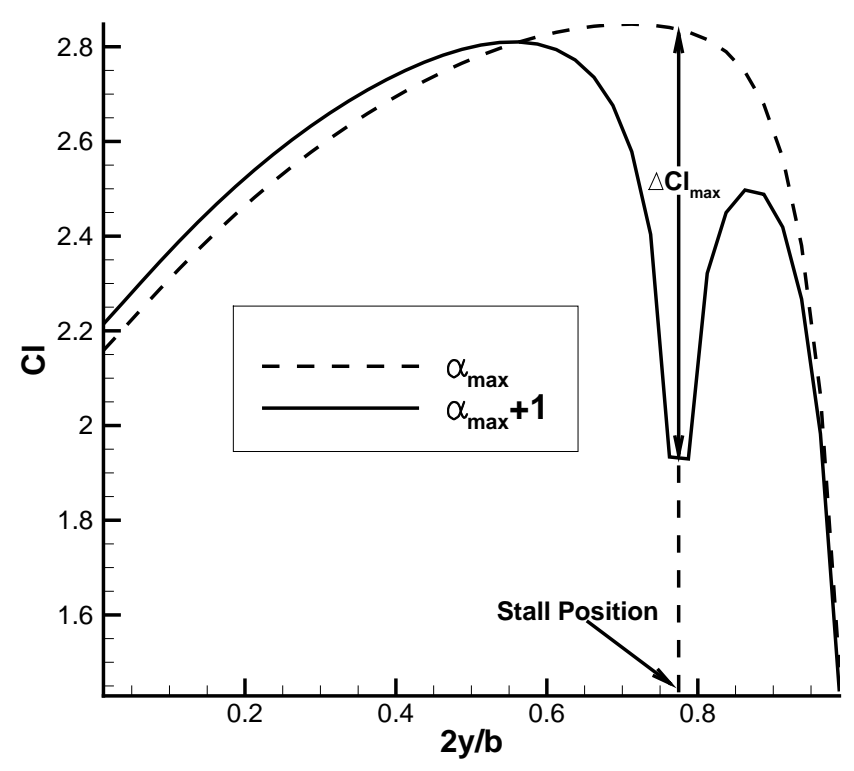

Figure 7: Spanwise stall detection scheme.

\section{Optimization Reference Geometry}

With the aerodynamic analysis tool set, we now proceed towards the optimization problem. The Lovell wing[22] is a $30.5^{\circ}$ leading edge swept wing (Figure 8) that was used for wind tunnel investigation on the effect of flap deflections. The geometry is simple with a constant airfoil geometry along the span and experimental results are available for the isolated wing with different flap deflections. For its simplicity and available geometry for clean and high-lift configurations, the isolated Lovell wing was chosen as the reference geometry for this optimization work. Note that the same wing was chosen by Valarezo and Chin to calibrate their stall model[21]. The VLM coupled with RANS solutions are compared against experimental data for the Lovell wing to verify that the aerodynamic coefficients are well estimated by the tool for the reference geometry. Figures 9, 10 and 11 present the overall aerodynamic coefficients for the clean configuration with different swept sectional data. A better maximum lift coefficient and pitching moment are obtained with the $30^{\circ}$ swept sectional data. The same observation is confirmed for the high-lift configuration with slat and flap deflected at $25^{\circ}$ and $10^{\circ}$ respectively (Figures 12 and 13). The numerical settings for the VLM model is to used 5 and 50 panels chordwise and spanwise, respectively. A verification of the model comparing 3D RANS solutions is made in reference [19]. 


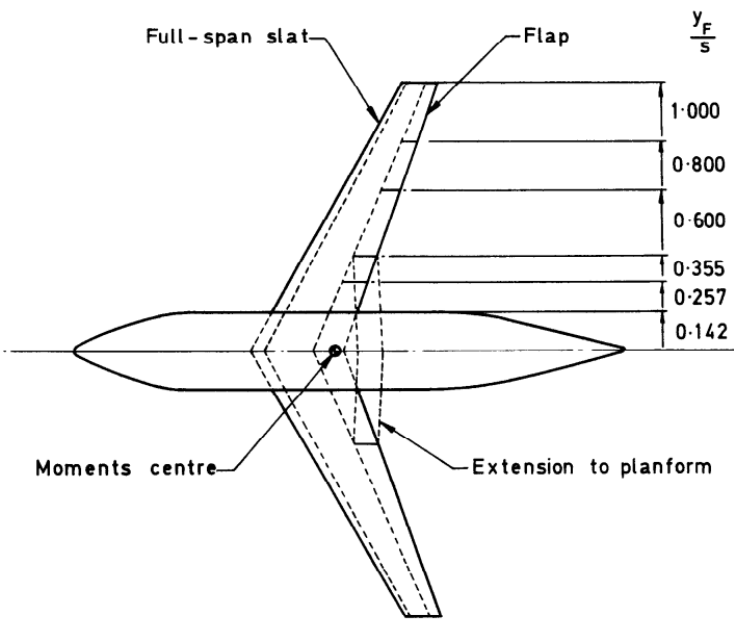

(a) Planform.

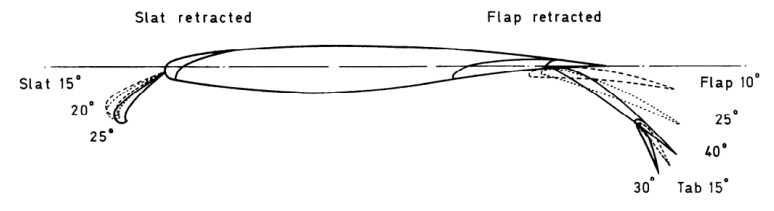

(b) Airfoil.

Figure 8: Lovell geometry[22].

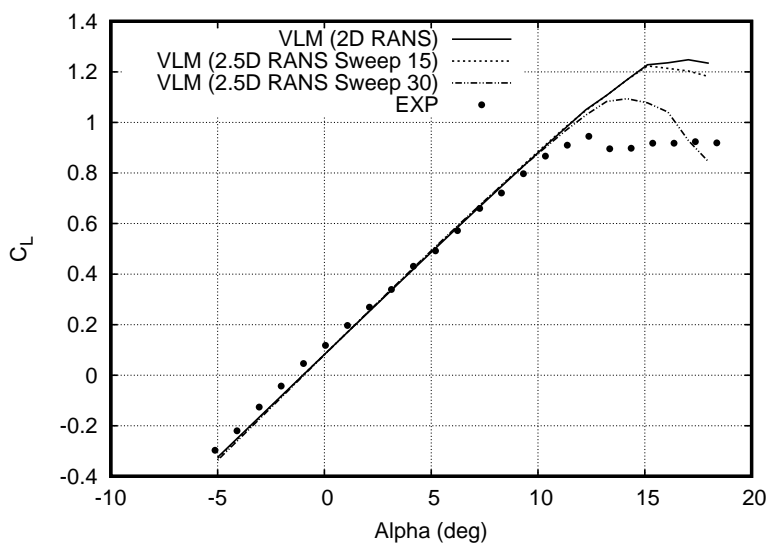

Figure 9: Lovell clean configuration $-C_{L}$.

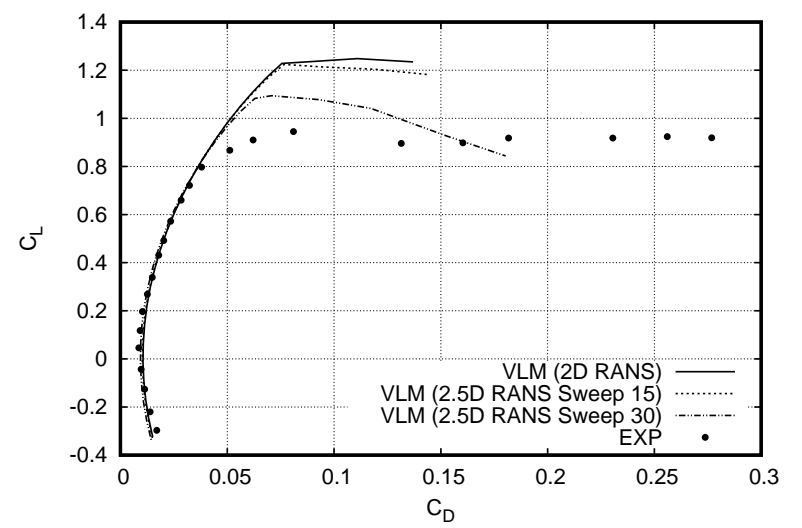

Figure 10: Lovell clean configuration $-C_{D}$.

\section{Evolution Strategy Optimizer}

The maximum lift coefficient and its position along the span are very sensitive in regards of the wing geometry, yielding a highly non-linear function, even non-continuous, which poses a problem for gradients evaluation. Therefore, Gradient free optimizers are preferable and a Covariance Matrix Adaptation Evolution Strategy (CMA-ES) was chosen for its well known performance on non-linear and non-continuous problems[12, 23]. CMA-ES is considered as state-of-the-art in evolutionary algorithm and reliable for global optimization[24]. Furthermore, CMA-ES requires almost no calibration except population size. The code is available from https://www.lri.fr/ hansen/cmaes_inmatlab.html and the Python version was used to be fully integrated inside the VLM C++ Python interface. 


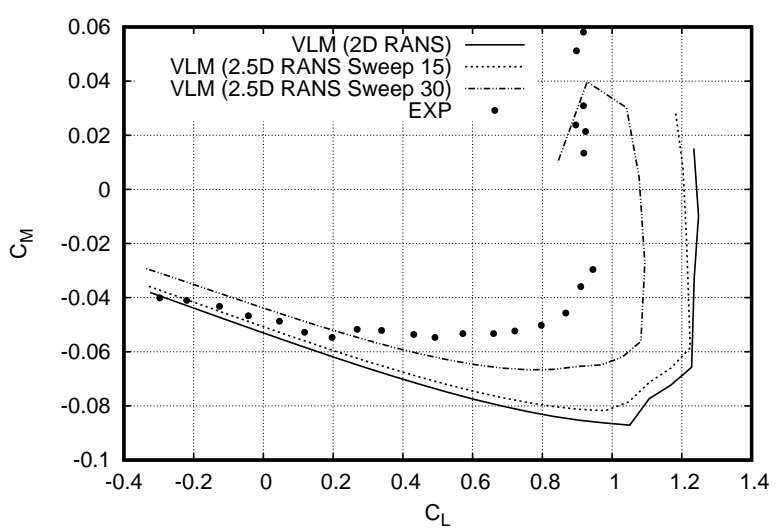

Figure 11: Lovell clean configuration $-C_{M}$.

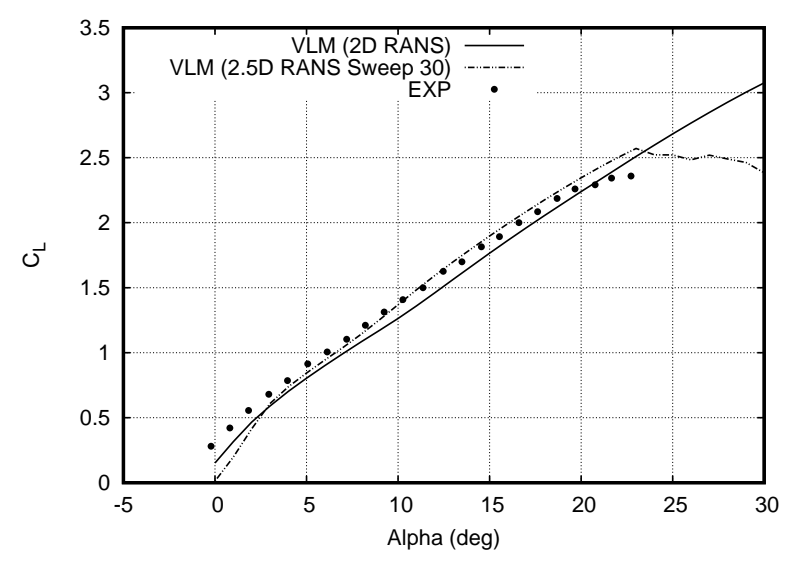

Figure 12: Lovell slat: $25^{\circ}$ flap: $10^{\circ}-C_{L}$.

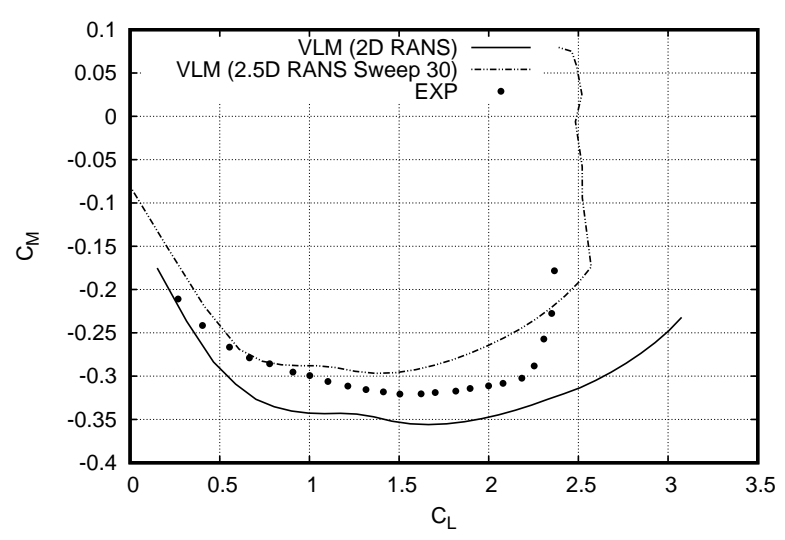

Figure 13: Lovell slat: $25^{\circ}$ flap: $10^{\circ}-\mathrm{C}_{M}$.

\subsection{Non-Linear Constraints}

CMA-ES does not handle constraints by default. However, a penalty constraint violation can be incorporated[25]. For every constraint $i$ and for a given solution $x$, the constraint value is computed as follows

$$
\gamma_{i}(x)=\frac{1}{\epsilon_{i}} \times\left\{g_{i}(x)+\epsilon_{i} \text { for inequality constraint } g_{i}(x) \leq 0.0\right\}
$$

where $\epsilon_{i}$ are user-defined values. When $\gamma_{i}>0$, the constraint is considered active and a penalty is added to the objective function. The penalty is evaluated with equation (19)

$$
f_{i}(x)= \begin{cases}w_{i} \gamma_{i}(x)^{2}, & \text { if } \gamma_{i}>0 \\ 0, & \text { otherwise }\end{cases}
$$

where $w_{i}$ are user-defined constants. Every penalty $f_{i}$ is added to the cost function. 


\subsection{VLM with CMA-ES Implementation Verification}

To verify the implementation of CMA-ES with the VLM, an induced drag minimization is performed with the twist as the design variable. The geometry used is the Lovell wing discretized with 50 panels spanwise and 5 panels chordwise (Figure 14). The twist is defined at 5 equally distant control sections along the span, thus 5 design variables are used. An inequality constraint for the lift coefficient, $C_{L} \geq 0.5$, is also added to verify the cost penalty implementation. Since the VLM is based on Prandtl's lifting-line theory, the optimal solution should be at $C_{L}=0.5$ with a twist distribution giving an elliptical spanload, thus the lowest induced drag.

Results show that an elliptical spanload is recovered by the optimizer as expected (Figure 15). Only 1000 function evaluations are necessary to achieve the optimal solution (Figure 16) which took under 20 minutes to complete on 6 cpus of intel 3930k. Furthermore, the inequality constraint on $C_{L}$ is handled properly with the final solution lying at $C_{L}=0.5$ as expected. These results confirms the implementation of CMA-ES with the VLM and the cost penalty constraint.

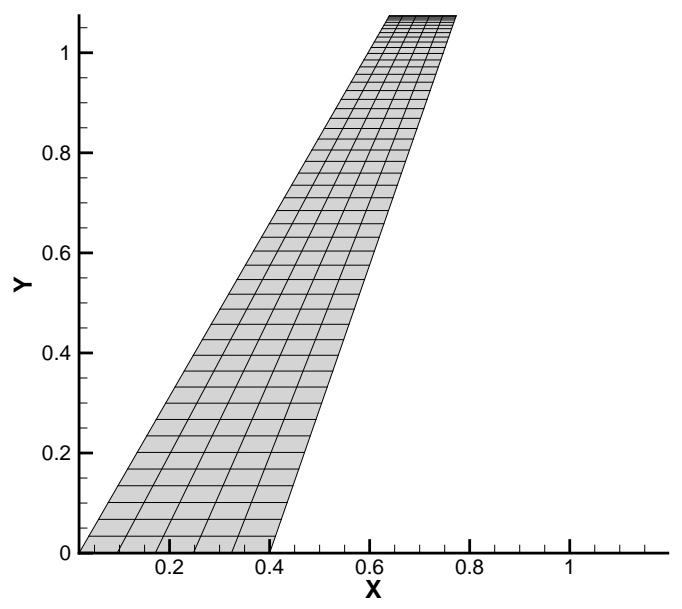

Figure 14: Lovell - VLM planform mesh. 


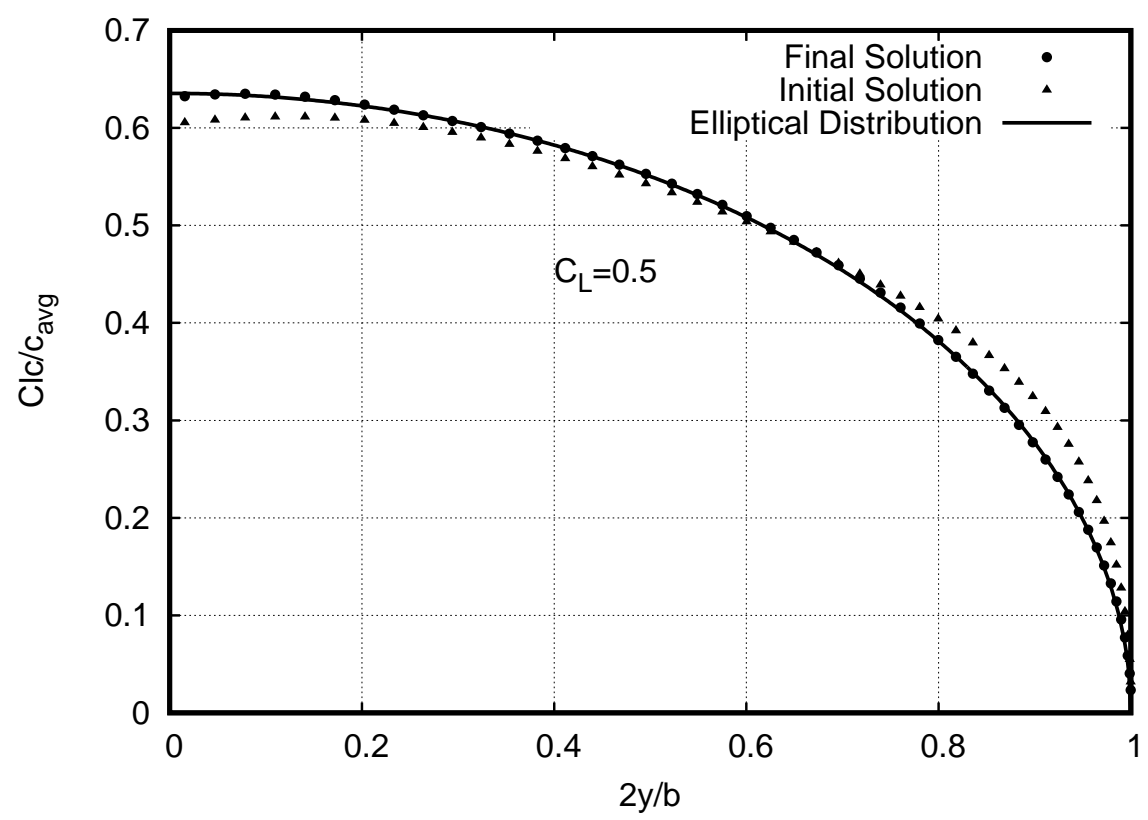

Figure 15: Twist optimization to minimize induced drag.

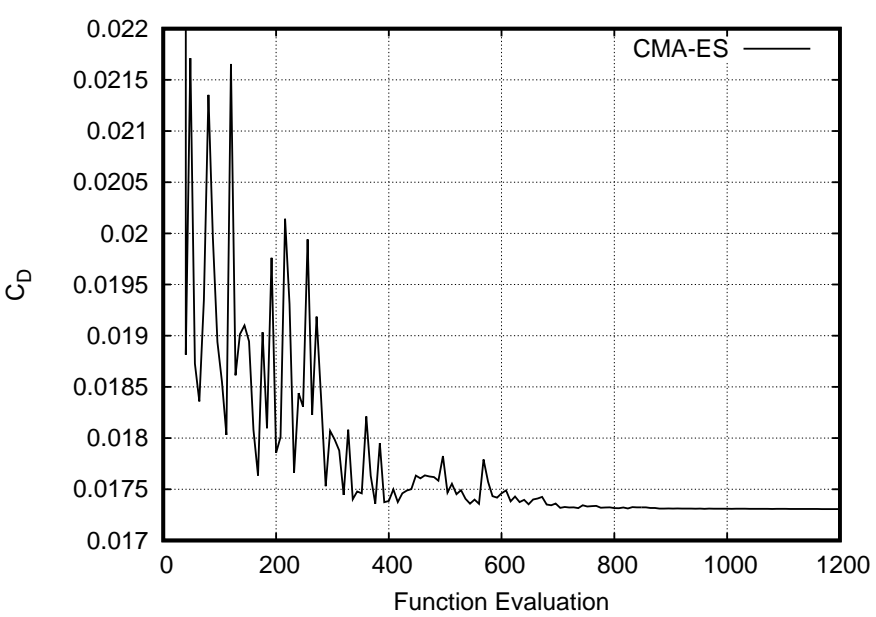

(a) Objective function convergence.

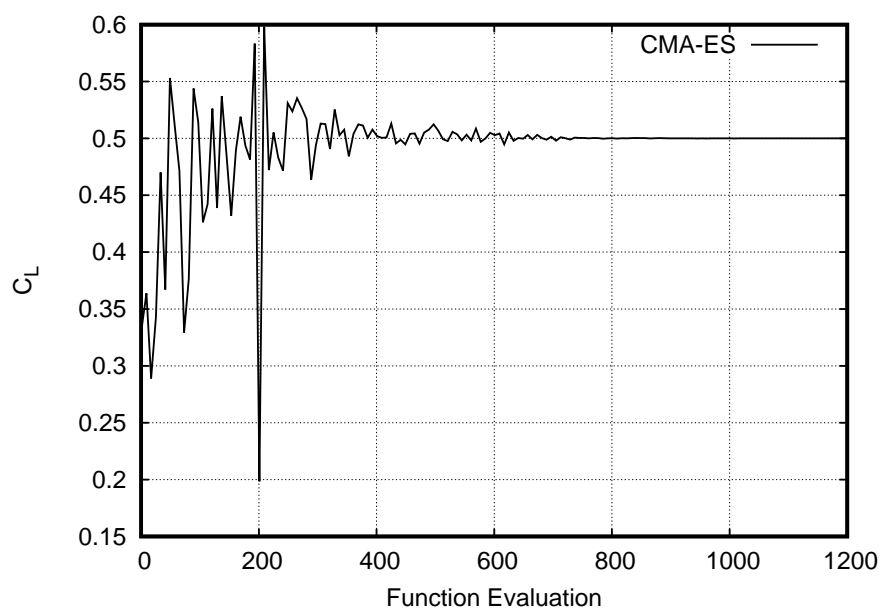

(b) Constraint $C_{L} \geq 0.5$.

Figure 16: CMAES - Induced drag optimization.

\section{Structural Model}

A structural constraint must be considered to avoid optimal solutions with unrealistic geometries, like infinite aspect ratio to reduce the induced drag. A solution is to incorporate the wing weight as a constraint, where the optimal solution cannot be heavier than the Lovell reference wing. Since the constraint is applied as a difference in terms of weight between the new configuration and the reference Lovell wing, the sensitivity with regards to the wing geometry is more important than the precision of the wing weight calculation. 
A sensitivity analysis was performed by Mariens[26] for different statistical methods for wing weight estimations and the results of the analysis are presented in Table 1. From the analysis, Torenbeek and EMWET are the two methods with the largest sensitivity with regards to wing parameters like sweep, thickness, span, etc... The Torenbeek weight estimation used is a statistical method[27] derived from a more complex quasi-analytical method derived also from Torenbeek. EMWET is a quasi-analytical tool developed by Elham[28] to estimate the wing weight. However, its implementation is more complex than Torenbeek's statistical approach and the structural accuracy is not the goal of the present work. Therefore, Torrenbeek was chosen to evaluate the wing weight, with the relation given by Equation 20.

\begin{tabular}{lccccccccc}
\hline Method & $W_{t o}$ & $W_{z f}$ & $b$ & $c_{r}$ & $\Lambda$ & $\lambda$ & $t / c_{r}$ & $t / c_{k}$ & $t / c_{t}$ \\
\hline \hline Torenbeek & $\checkmark$ & $\checkmark$ & $\checkmark$ & $\checkmark$ & $\checkmark$ & $\checkmark$ & $\checkmark$ & $\checkmark$ & $\checkmark$ \\
Howe & $\checkmark$ & & $\checkmark$ & & $\checkmark$ & $\checkmark$ & $\checkmark$ & & \\
EMWET & $\checkmark$ & $\checkmark$ & $\checkmark$ & $\checkmark$ & $\checkmark$ & $\checkmark$ & $\checkmark$ & $\checkmark$ & $\checkmark$ \\
Shevell & $\checkmark$ & & $\checkmark$ & $\checkmark$ & $\checkmark$ & $\checkmark$ & $\checkmark$ & $\checkmark$ & $\checkmark$ \\
LTH & $\checkmark$ & & $\checkmark$ & $\checkmark$ & $\checkmark$ & $\checkmark$ & $\checkmark$ & $\checkmark$ & $\checkmark$ \\
\hline
\end{tabular}

Table 1: Wing weight estimation method sensitivity to wing parameters (Adapted from [26]).

$$
W_{w}=0.06 \frac{w}{\sigma_{r}} n_{u l t} \sqrt{W_{t o} W_{z f}} \frac{b^{3}}{S} \frac{1}{(t / c)_{a v e} \cos ^{2} \Lambda} \frac{1+2 \lambda}{1+\lambda}+w t_{s s} S
$$

\section{Sectional Viscous Database and Design Variables}

This section explores the optimization problem complexity that can be achieved using 2.5D RANS data with the non-linear VLM approach. Since low-speed $C_{L_{\max }}$ and transonic cruise conditions are considered, the RANS sectional data must be generated with great care and for a wide range of conditions to perform a full flight spectrum optimization. At low-speed conditions, RANS simulations were performed for the clean configuration and for high-lift configuration with slat and flap deflected for three different flap deflections $\left(10^{\circ}\right.$, $\left.25^{\circ}, 40^{\circ}\right)$. For cruise conditions, only the clean configuration was considered at four different Mach numbers $(0.6,0.7,0.75,0.8)$.

All the simulations were also performed at three different Reynolds numbers to capture the effect of taper ratio. Finally, to incorporate the viscous effects of crossflow over swept wings, every simulation was performed at nine different sweep angles $\left(0^{\circ}, 5^{\circ}, 10^{\circ}, 15^{\circ}\right.$, $20^{\circ}, 25^{\circ}, 30^{\circ}, 35^{\circ}, 40^{\circ}$ ) with the 2.5D RANS flow solver NSCODE[15] using the SpalartAllmaras turbulence model. More than 3000 2.5D RANS calculations were necessary to generate the database. These computations are performed only once and can be used for any optimization problem. Afterwards, linear interpolation is used to generate the appropriate viscous database during the optimization, making for a rapid function evaluation. The viscous database is summarized in Table 2. Note that standard CFD practices are used in 
generating the RANS solutions: farfield located at 100 chord; $y^{+}<1$; residuals are converged to $10^{-} 5$ and the mesh used is fine enough to be considered mesh-independent within the tolerance required. More details on the mesh generation process used is described in [29] with the flow solver accuracy presented in Pigeon[15].

Planform optimization is considered in the present study with seven design variables defining the geometry (Table 3 and Figure 17). The wing twist is defined by a linear function fixed by the root and tip twist. The tip chord is given a lower bound of 0.13 to prevent unrealistic geometries and ensure sufficient chord length for winglets. The leading edge sweep is bound between $0^{\circ}$ to $40^{\circ}$ which corresponds to the range of sweep evaluated in the viscous database. The spanwise kink position is set to a minimum of 0.1524 that corresponds to the actual Lovell wing kink position and the chord length at the kink position is controlled by the trailing edge sweep. The root chord is given a lower bound of 0.2 to avoid again unrealistic geometries. The planform surface area is also kept constant to the Lovell reference geometry, thus the span is evaluated at each function evaluation with the design variables to keep the wing surface area constant.

\begin{tabular}{lcc}
\hline & Low-Speed & High-Speed \\
\hline \hline Mach Number & 0.2 & $0.6,0.7,0.75,0.8$ \\
Reynold's Number & $0.7 \mathrm{E} 6,1.3 \mathrm{E} 6,2 \mathrm{E} 6$ & $3.3 \mathrm{E} 6,6.3 \mathrm{E} 6,9.3 \mathrm{E} 6$ \\
Sweep & $0,5,10,15,20,25,30,35,40$ \\
Angle of Attack & \multicolumn{2}{c}{-6 to Post-Stall } \\
Slat & Retracted, Deflected: 25 & Retracted \\
Flap & Retracted, Deflected: $10,25,40$ & Retracted \\
\hline
\end{tabular}

Table 2: Viscous database.

\begin{tabular}{lcc}
\hline \multicolumn{3}{c}{ DESIGN VARIABLES (7) } \\
\hline \hline & Lower Bound & Upper Bound \\
Root Chord (V1) & 0.2 & - \\
TE Kink Sweep (V2) & 0 & 90 \\
Tip Chord (V3) & 0.13 & - \\
LE Sweep (V4) & 0 & 40 \\
Root Twist (V5) & -10 & 10 \\
Tip Twist (V6) & -10 & 10 \\
Kink Position (V7) & 0.1524 & - \\
\hline Wing Surf. Area & \multicolumn{2}{c}{0.5523 (Constant) } \\
Span & Calculated from the design variables \\
\hline
\end{tabular}

Table 3: Design variables for planform optimization. 


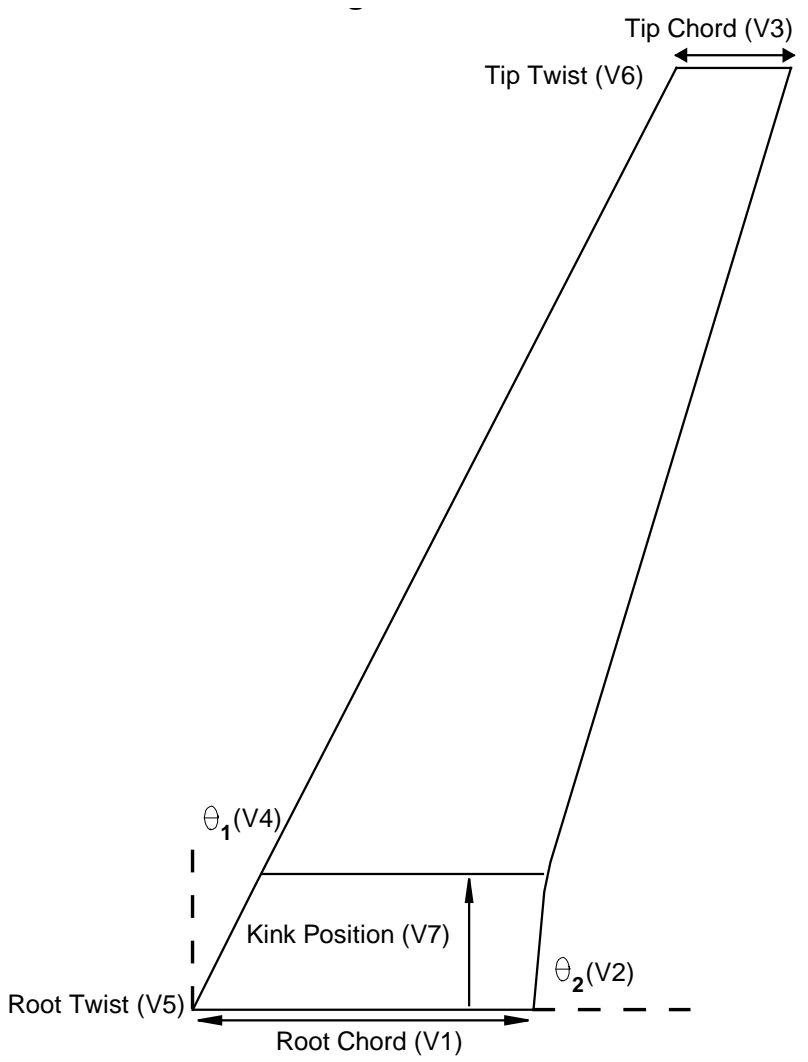

Figure 17: Design variables for planform optimization.

\section{Optimization Results}

\subsection{High-Speed Optimization}

The use of 2.5D RANS sectional data has a significant effect on the transonic shock and allows cruise optimization with wave drag sensitivity to sweep. The same optimization is performed for different cruise Mach numbers to evaluate compressibility effects over the optimal planform. Since the twist is a design variable, the angle of attack is fixed at $1^{\circ}$. The optimization problem is defined as follow,

$$
\begin{array}{ll}
\underset{x}{\operatorname{minimize}} & -\frac{C_{L}}{C_{D}} \\
\text { subject to } & W_{w}(x)-W_{w_{\text {reference }}} \leq 0.0
\end{array}
$$

where $W$ is the wing weight calculated with Torenbeek (Equation 20). The weight constraint ensures the solution is not heavier than the reference Lovell wing.

As the cruise Mach number is increased, the optimal planform sweep is also increased (Figure 18) from $22.16^{\circ}$ to $37.91^{\circ}$ LE sweep. At a high Mach number, increasing sweep reduces wave drag, thus the optimal solution at a Mach number of 0.8 has a higher LE sweep of $37.91^{\circ}$ compared to the Lovell wing $30.5^{\circ}$ LE sweep. Therefore, the optimized planform has lower viscous drag (Figure 19) which comprise drag due to shocks. These 
results confirm the sensitivity of viscous drag with regard to sweep from the 2.5D RANS sectional data.

Additionally, the optimizer has successfully brought the maximum lift-to-drag ratio at the desired angle of attack of $1^{\circ}$ (Figures 20-21). CMA-ES also managed to keep roughly the same lift-to-drag ratio around 29 for the different cruise Mach numbers (Table 4). The optimal lift coefficient at $1^{\circ}$ is also lower with increasing Mach number.

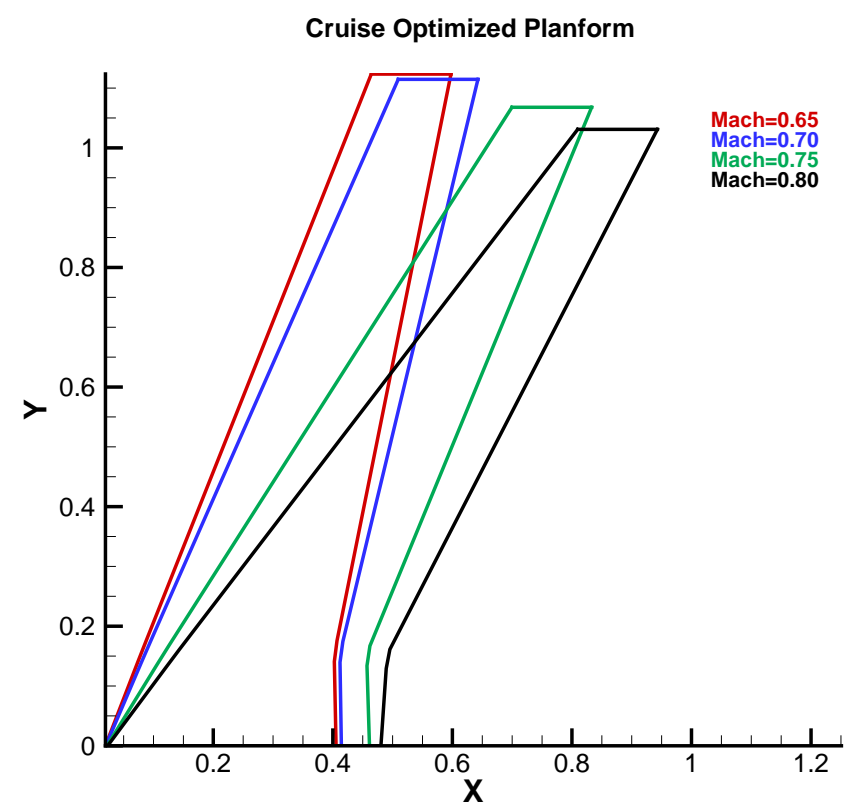

Figure 18: Optimized planform for different cruise Mach number.

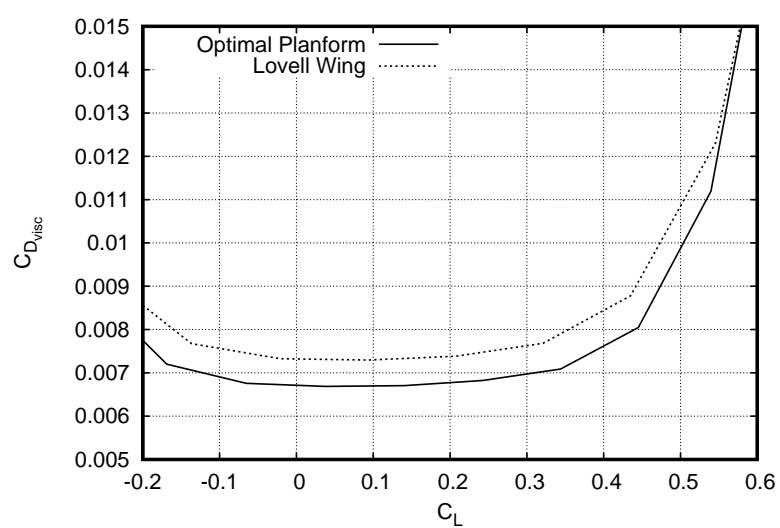

Figure 19: Viscous drag vs $C_{L}$ at Mach $=0.8$.

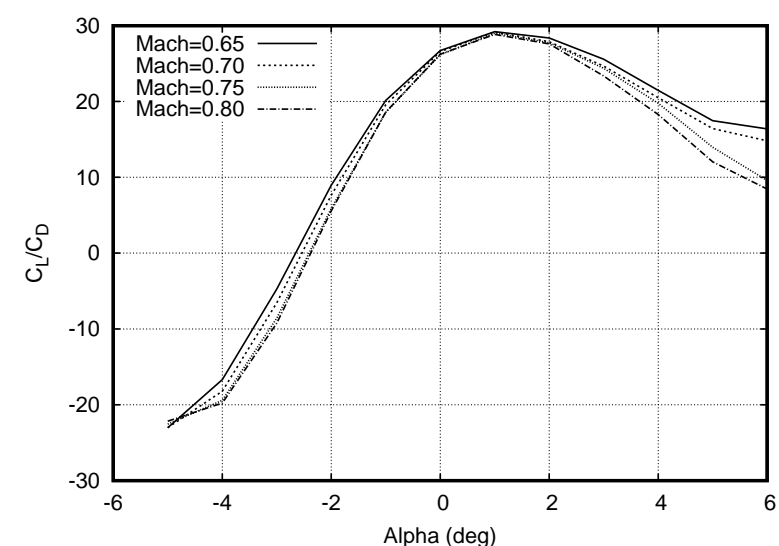

Figure 20: Lift-to-drag Ratio of optimized planform for different cruise Mach number. 

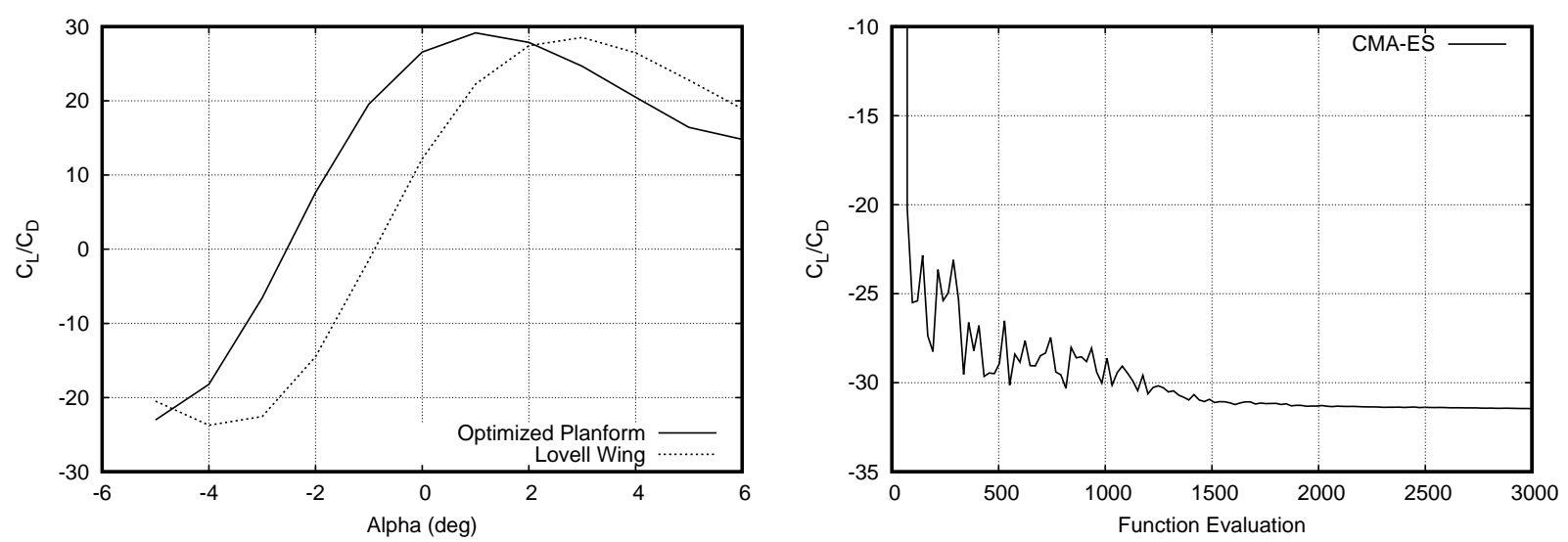

Figure 21: Lift-to-drag ratio of optimized planform Figure 22: Lift-to-drag ratio optimization converat $\mathrm{Mach}=0.7$. gence at $M a c h=0.75$.

\begin{tabular}{lcccccc}
\hline Mach & $\mathbf{0 . 6}$ & $\mathbf{0 . 6 5}$ & $\mathbf{0 . 7}$ & $\mathbf{0 . 7 5}$ & $\mathbf{0 . 7 8}$ & $\mathbf{0 . 8}$ \\
\hline \hline CL & 0.409 & 0.394 & 0.391 & 0.359 & 0.333 & 0.344 \\
CL/CD & 29.36 & 29.11 & 29.11 & 28.95 & 28.71 & 28.85 \\
\hline
\end{tabular}

Table 4: Optimized results for different Mach number at $\alpha=1^{\circ}$.

\subsection{Low-Speed Optimization}

The aerodynamic design of high-lift systems requires a trade-off between maximum lift coefficient and lift-to-drag ratio. The takeoff distance and the climb performance are strongly affected by lift-to-drag ratio. On the other hand, the required approach airspeed for airworthiness[3] must be $1.23 \times V_{s_{1 g}}$ where $V_{s}$ is the stall speed. Therefore landing performance is directly related to $C_{L_{\max }}$. Another important airspeed at takeoff is $V_{2}$, the speed at which the aircraft may safely climb with one engine inoperative, $V_{2}=1.13 \times V_{s}$ [3]. The lift-to-drag ratio at $V_{2}$ influences directly climb performance and $V_{2}$ can also be expressed in terms of lift coefficient, $C_{L_{2}}=\frac{C_{L_{\max }}}{1.13^{2}}$. The lift-to-drag ratio at $C_{L_{2}}$ should therefore be considered as an objective function with $C_{L_{\max }}$. However, CMA-ES is a single objective optimizer, thus composite objective function with user-defined weights is used (Equation 21). For simplicity, the high-lift configuration is considered constant along the span with the slat deflected at $25^{\circ}$ and the flap deflected at $10^{\circ}$.

Single objective $C_{L_{\max }}$ optimization yields a solution with a low lift-to-drag ratio of 7.76 at $C_{L_{2}}$ and a lower aspect ratio planform of 7.42 (Figure 23 and Table 5). Adding the lift-to-drag ratio to the objective function is necessary for takeoff performance. Thus, one function evaluation goes as follows:

1. Find $C_{L_{\max }}$ by incrementing the angle of attack until the lift curve slope becomes negative.

2. Calculate $C_{L_{2}}=\frac{C_{L_{\max }}}{1.13^{2}}$.

3. Calculate $C_{D}$ at $C_{L_{2}}$ with a Proportional Integral Derivative controller[30] over the angle of attack for an accurate evaluation of $C_{D}$ at $C_{L_{2}}$. 
The tradeoff between climb performances and $C_{L_{\max }}$ is observed by a pareto front when performing the same optimization with different weights for $C_{L_{\max }}$ (Figure 24). As the weight is reduced for $C_{L_{\max }}$ in the objective function, the optimal planform sweep is increased, which in turns reduces the $C_{L_{\max }}$ and increases the lift-to-drag ratio at $C_{L_{2}}$ (Figure 25 and Table $5)$.

$$
\begin{array}{ll}
\underset{x}{\operatorname{minimize}} & -W_{1}\left(C_{L_{\max }}\right)-W_{2}\left(\frac{C_{L}}{C_{D}}\right)_{@ C_{L_{2}}} \\
\text { subject to } & W_{w}(x)-W_{w_{\text {reference }}} \leq 0.0
\end{array}
$$

Optimized Planform

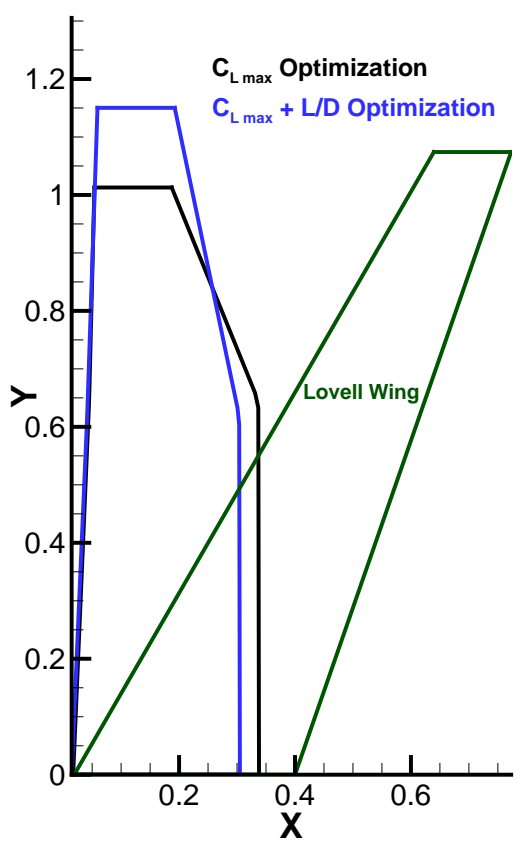

Figure 23: $\left(C_{L_{\max }}\right)$ Optimization compared with $\left(C_{L_{\max }}+\frac{C_{L}}{C_{D}}\right.$ at $\left.C_{L_{2}}\right)$ optimization.

\subsubsection{Spanwise Stall Constraint}

It is required per certification of transport aircraft that handling qualities must be adequate to allow a safe recovery from high angle of attacks where stall conditions are reached (Federal Aviation Administration[31]). However, swept tapered wings tend to have a higher spanload towards the tip which contributes to a less stable outboard stall. The VLM/RANS solver allows the spanwise stall position to be detected with stall cells[20] (Figure 7) and can be introduced as a constraint in the optimization to ensure appropriate stall conditions. The spanwise stall constraint is chosen to be less than $60 \%$ of the span.

The stall constraint is well handled by the optimizer (Figure 26) with the optimal planform stalling at $58 \%$. Moreover, adding the stall constraint has a negligible effect on the 

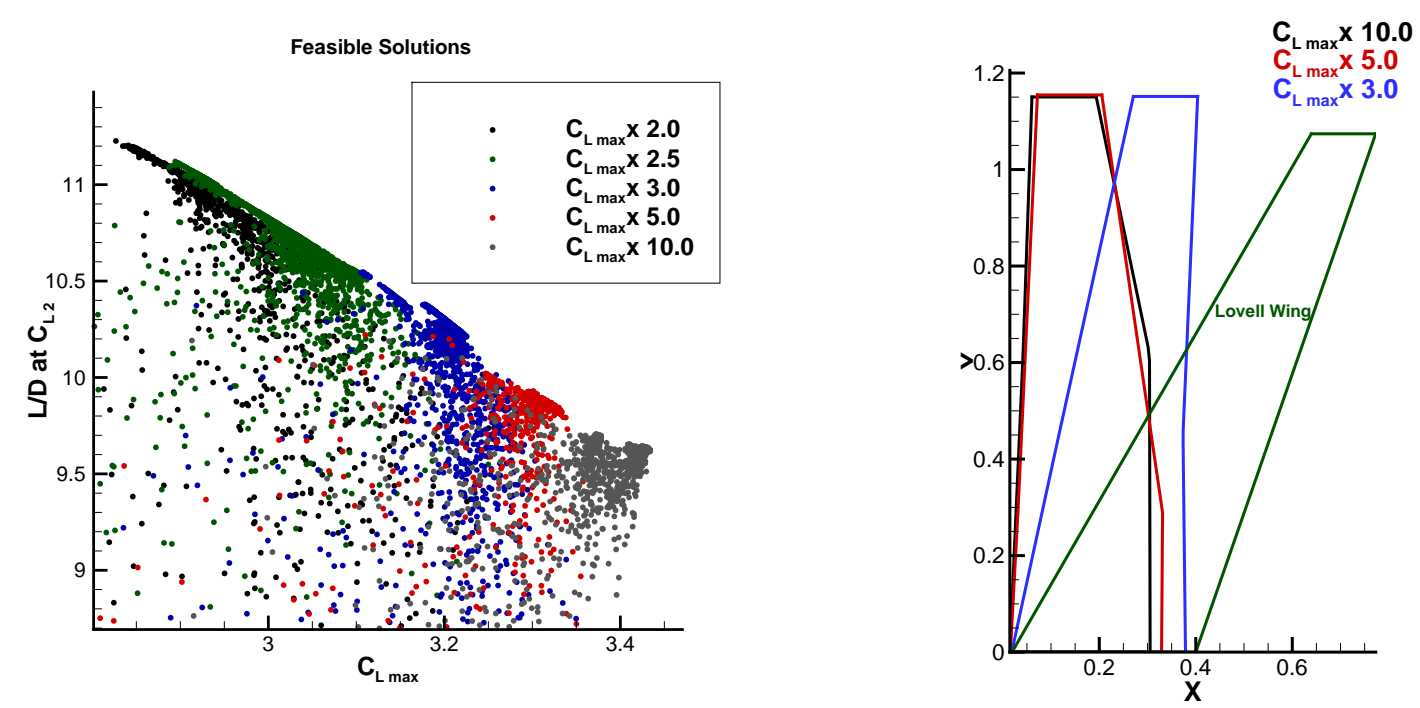

Figure 24: Optimization solution with different weights for $C_{L_{\max }}$.

Figure 25: Optimization solution comparison with different weights for $C_{L_{\max }}$.

\begin{tabular}{|lccc|}
\hline & Weights & $C_{L_{\max }}$ & $\frac{C_{L}}{C_{D}}$ \\
\hline$W_{C_{L_{\max }}}$ & 1.0 & 3.45 & 7.76 \\
$W_{\frac{C_{L}}{C_{D}}}$ & 0.0 & & \\
\hline$W_{C_{L_{\max }}}$ & 2.0 & 2.85 & 11.19 \\
$W_{\frac{C_{L}}{C_{D}}}$ & 1.0 & & \\
\hline$W_{C_{L_{\max }}}$ & 2.5 & 2.89 & 11.12 \\
$W_{\frac{C_{L}}{C_{D}}}$ & 1.0 & & \\
\hline$W_{C_{L_{\max }}}$ & 3.0 & 3.18 & 10.37 \\
$W_{\frac{C_{L}}{C_{D}}}$ & 1.0 & & \\
\hline$W_{C_{L_{\max }}}$ & 5.0 & \multirow{2}{*}{3.32} & 9.84 \\
$W_{\frac{C_{L}}{C_{D}}}$ & 1.0 & & \\
\hline$W_{C_{L_{\max }}}$ & 10.0 & 3.43 & 9.61 \\
$W_{\frac{C_{L}}{C_{D}}}$ & 1.0 & & \\
\hline
\end{tabular}

Table 5: Low-Speed optimization results.

overall planform (Figure 27), but does have important effects on the twist distribution (Table 6) with a $4.02^{\circ}$ root twist and $-1.55^{\circ}$ tip twist. 


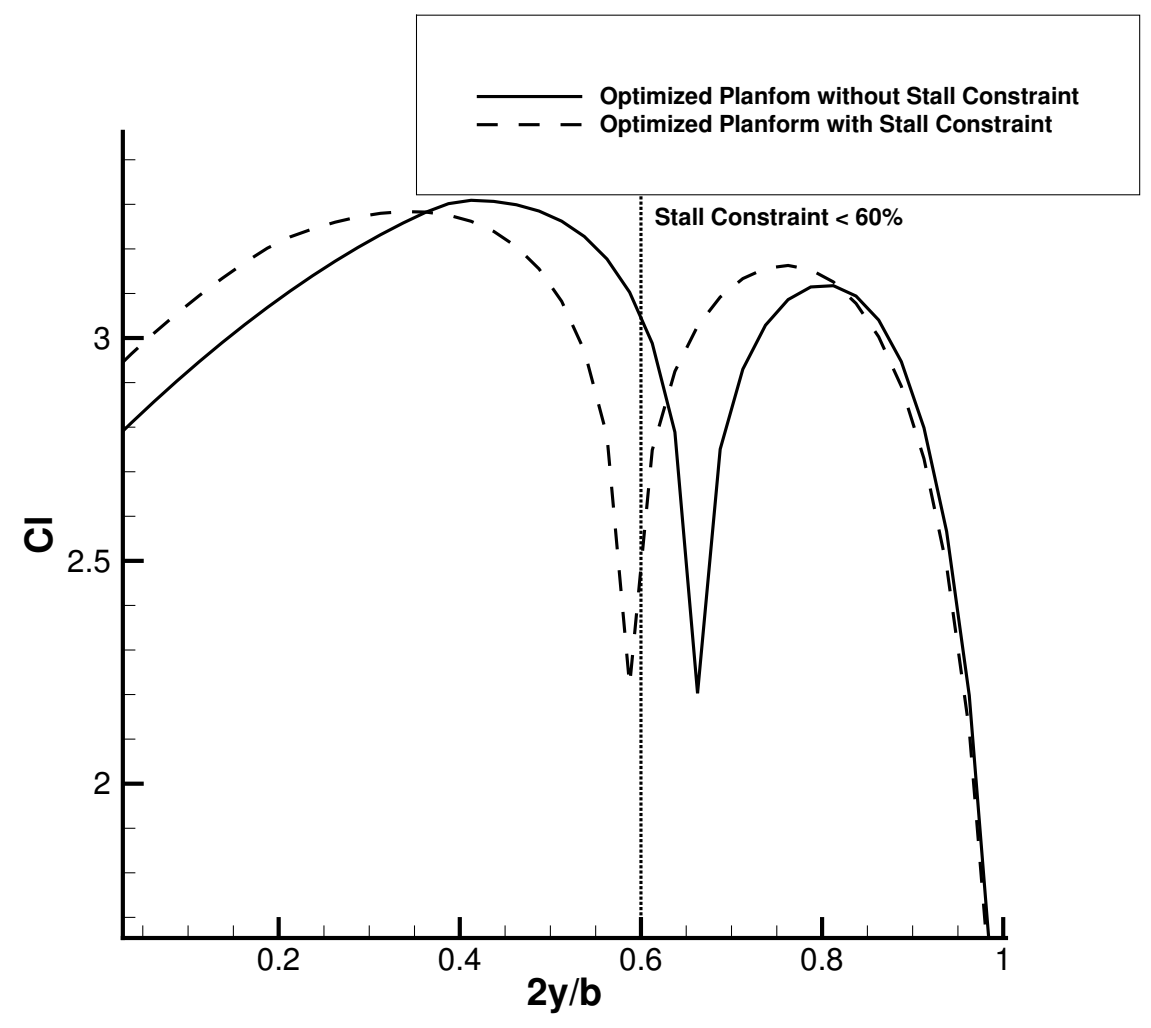

Figure 26: Spanwise stall constraint.

\begin{tabular}{lccccc}
\hline & Root Twist & Tip Twist & $\boldsymbol{C}_{\boldsymbol{L}_{\max }}$ & $\frac{\boldsymbol{C}_{L}}{\boldsymbol{C}_{D}}$ & Spanwise Stall Pos. \\
\hline \hline $\begin{array}{l}\text { Optimization without } \\
\begin{array}{l}\text { Stall Constraint } \\
\text { Optimization with Stall }\end{array}\end{array}$ & -3.06 & -1.66 & 3.18 & 10.37 & $67 \%$ \\
Constraint & 4.02 & -1.55 & 3.24 & 10.14 & $58 \%$ \\
\hline
\end{tabular}

Table 6: Optimal solution with and without spanwise stall constraint. 

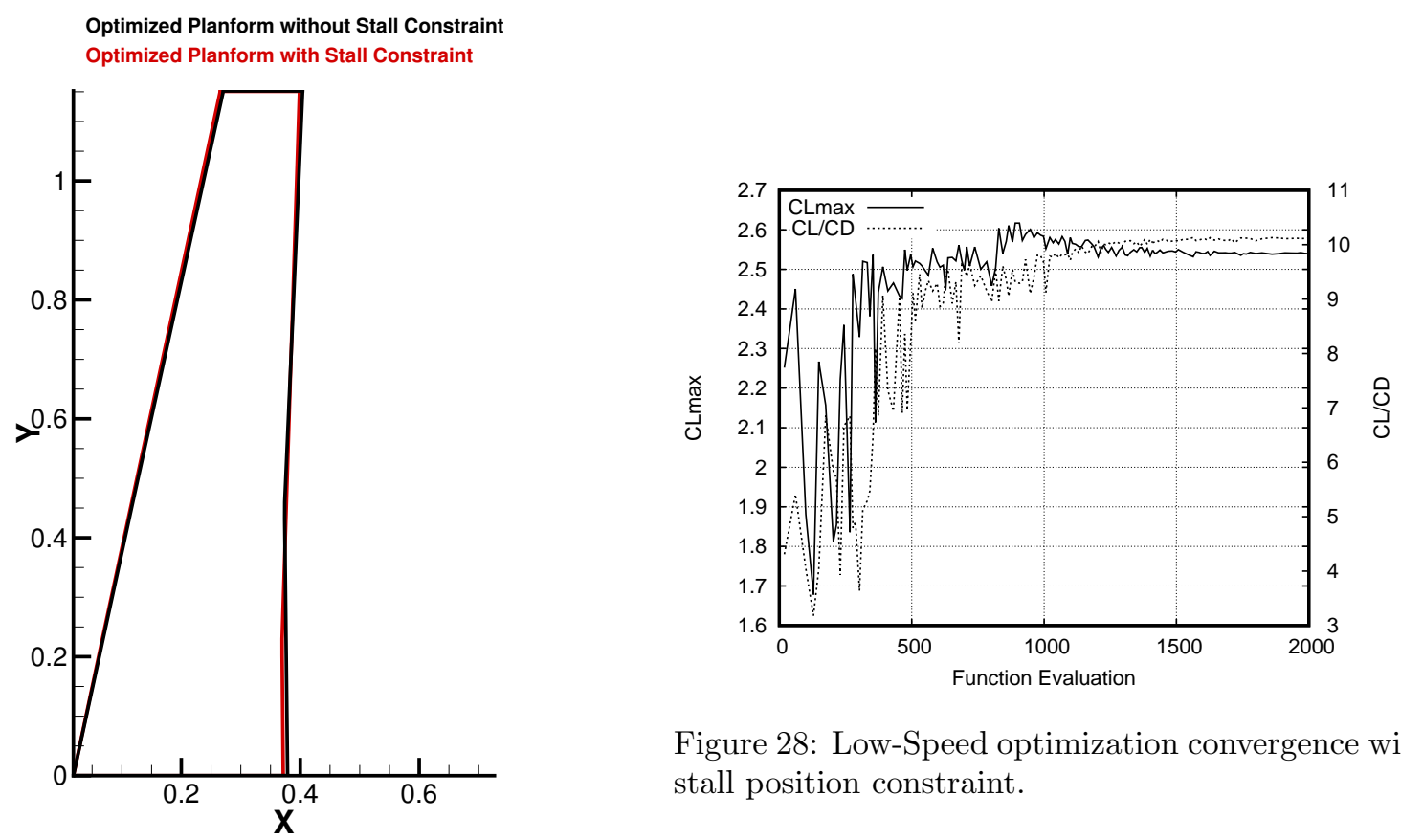

Figure 28: Low-Speed optimization convergence with stall position constraint.

Figure 27: Spanwise stall constraint optimized planform. 


\subsubsection{High-Lift Configuration Optimization}

Since the clean planform is modeled by the VLM while the section characteristics are incorporated by the viscous data, multi-topology high-lift optimization can be performed by assigning different viscous sectional data corresponding to different high-lift configurations along the span. One scenario explored is a slat optimization to achieve a minimum $C_{L_{\max }}$. The problem is defined with 2 design variables, the start and end points of the slat along the span. The objective function is to minimize the slat length for a required $C_{L_{\max }}$ (Equation $22)$.

The optimal solution for different $C_{L_{\max }}$ constraint (Figure 29) shows the continuity of the solution, thus confirming the performance and robustness of CMA-ES and the aerodynamic solver. One interesting finding is that the optimizer does not extend the slat to $100 \%$ of the span, as the tip-region local AoA is below stall at the wing $C_{L_{\max }}$ due to strong velocities induced by tip vortex.

In particular, it does not protect the wing tip, as the local angle of attack is below stall at the wing $C_{L_{\max }}$.

$$
\begin{array}{cl}
\underset{y_{1}, y_{2}}{\operatorname{minimize}} & L_{\text {slat }}\left(y_{1}, y_{2}\right)=y_{2}-y_{1} \\
\text { subject to } & C L_{\text {maxTarget }}-C L_{\max }\left(y_{1}, y_{2}\right) \leq 0.0 \\
& y_{1}-y_{2} \leq 0.0
\end{array}
$$


MINIMUM SLAT REQUIREMENT FOR A GIVEN CLmax
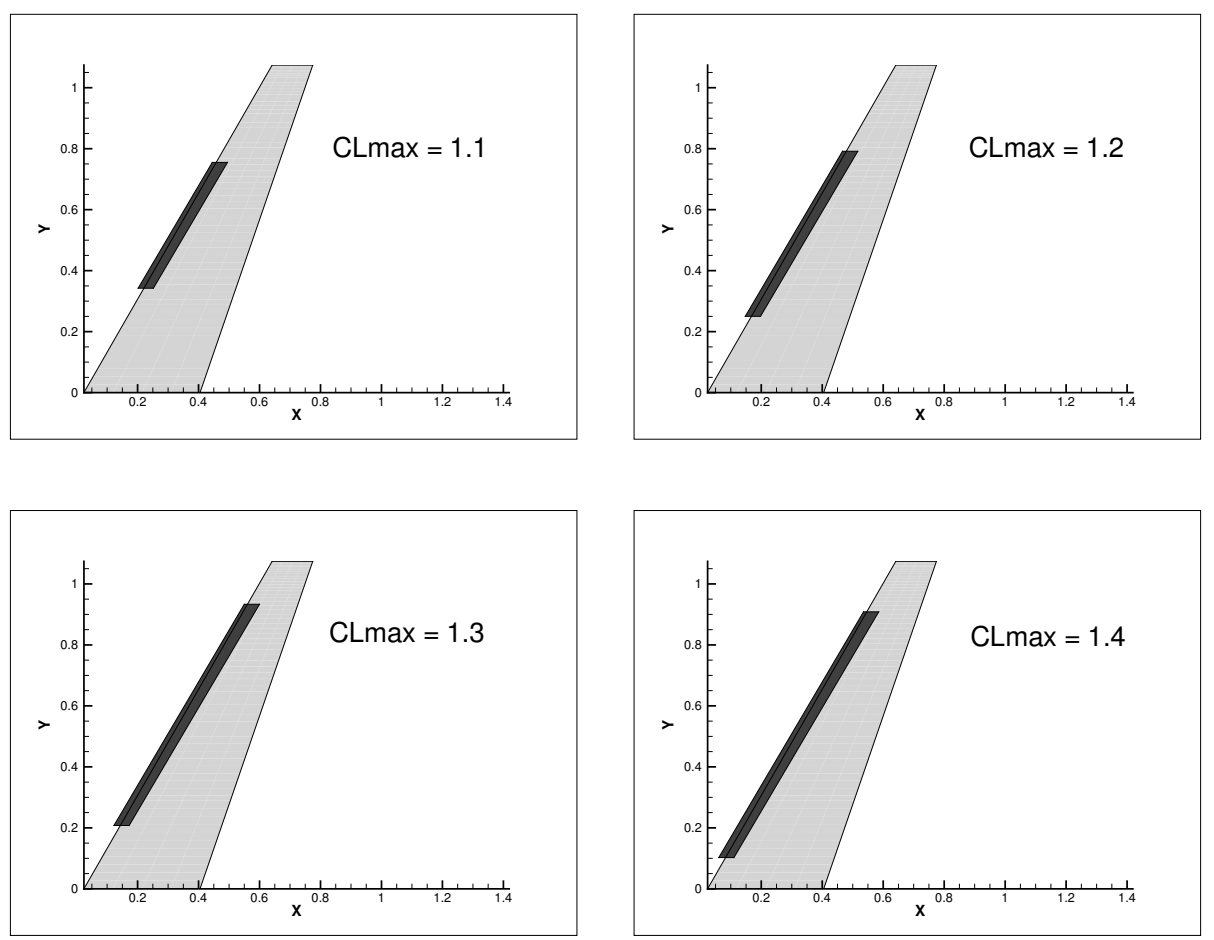

Figure 29: Optimization of slat configuration.

\subsection{Multi-Objective Low-Speed and High-Speed Optimization}

Finally, a composite objective function optimization combining the previous low-speed and high-speed objectives is explored with cruise conditions at Mach $=0.75$ (Equation 23). The optimal planform lies between the low-speed and high-speed optimal planforms with a moderate leading edge sweep of $17.8^{\circ}$ (Figure 30 ). The low-speed optimized planform has poor cruise performance with a lift-to-drag ratio of 9.74 (Table 7). On the other hand, the high-speed optimized planform has a relatively low $C_{L_{\max }}$ of 2.596 compared to the lowspeed optimized planform. Combining low-speed and high-speed objective functions result in a solution with good stall characteristics, climb performances and cruise performances as well (Table 7).

$$
\begin{array}{ll}
\underset{x}{\operatorname{minimize}} & -5.0 \times\left(C_{L_{\text {max }}}\right)-1.0 \times\left(\frac{C_{L}}{C_{D}}\right)_{@ C_{L_{2}}}-1.0 \times\left(\frac{C_{L}}{C_{D}}\right)_{\text {cruise }} \\
\text { subject to } & W_{w}(x)-W_{w_{\text {reference }}} \leq 0.0
\end{array}
$$




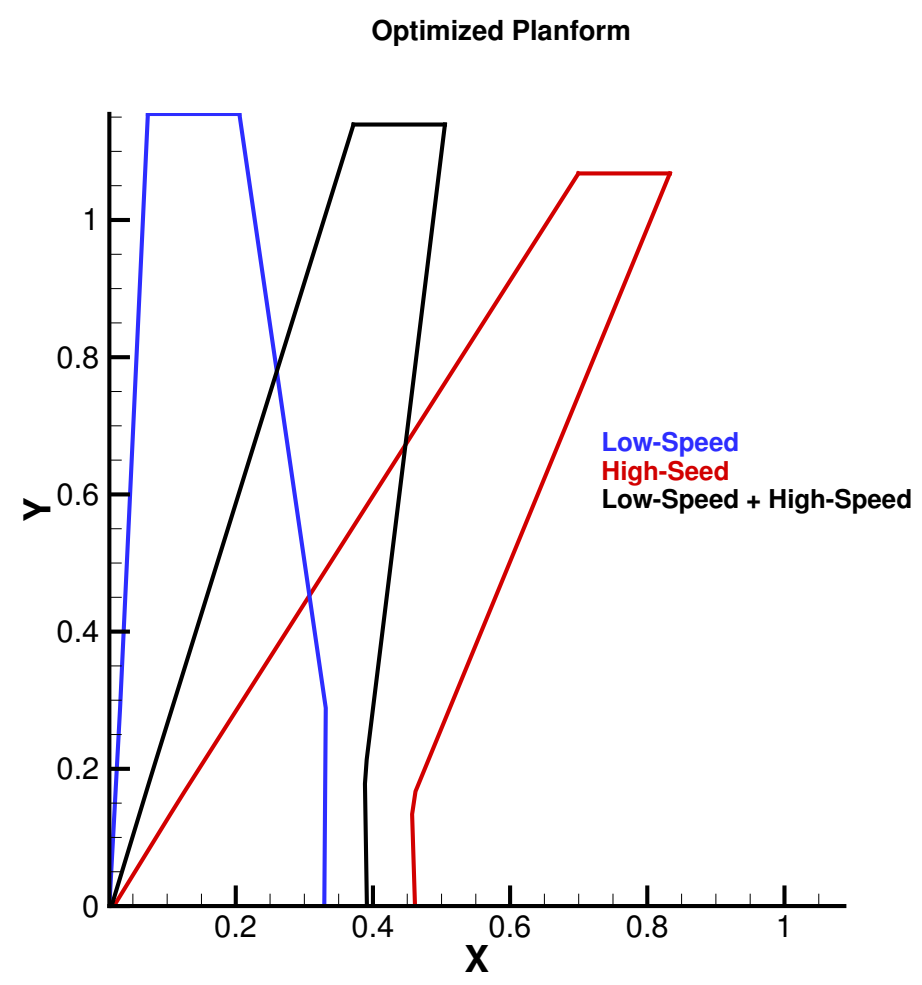

Figure 30: Optimized planform comparison with composite low-speed/high-speed objective function at Mach $=0.75$ for cruise conditions.

\begin{tabular}{lcccc}
\hline & $\boldsymbol{C}_{\boldsymbol{L}_{\text {cruise }}}$ & $\frac{\boldsymbol{C L}}{\boldsymbol{C D}} @ \boldsymbol{C}_{\boldsymbol{L}_{\text {cruise }}}$ & $\boldsymbol{C}_{\boldsymbol{L}_{\max }}$ & $\frac{\boldsymbol{C L}}{\boldsymbol{C D} @ \boldsymbol{V}_{\mathbf{2}}}$ \\
\hline \hline Low-Speed Optimization & 0.086 & 9.74 & 3.32 & 9.84 \\
High-Speed Optimization & 0.359 & 28.95 & 2.596 & 10.96 \\
Low-Speed/High-Speed Optimization & 0.46 & 27.02 & 3.12 & 10.48 \\
\hline
\end{tabular}

Table 7: High-speed and low-Speed optimization performed at Mach $=0.75$ for cruise conditions. 


\section{Conclusion}

A fast turnaround aerodynamic tool suitable for conceptual and preliminary design phase requirements is presented. High-speed and high-lift analyses are performed with a Vortex Lattice Method coupled to an infinite-swept wing RANS solver via a stripwise angle-of-attack correction procedure. A novel physics-based stall criteria is developed to obtain $C_{L_{\max }}, \alpha_{\max }$ and the spanwise location of the stall, a net improvement over the semi-empirical Valarezo method. Aerodynamic forces are directly obtained from the coupled VLM/RANS procedure, by spanwise integration of the RANS forces and Treffz-plane analysis of the VLM solutions. In particular, multi-element analysis is performed on the same mesh as the clean wing mesh. The analysis tools are embedded within a gradient-free Covariance Matrix Adaptation Evolution Strategy to perform aerodynamic optimization. The algorithm is verified on a canonical minimum induced-drag case and validated on several combined high-speed/highlift cases, including multi-topology optimisation of partial-span slat. The framework runs a single analysis within seconds on a single-CPU, allowing the use of gradient-free optimization strategies that are required during the early aircraft design phases.

\section{Acknowledgments}

This research is a collaborative effort between ONERA and École Polytechnique de Montréal and was made possible by a MITACS scholarship and support from Bombardier Aerospace.

\section{References}

[1] P. Piperni, A. DeBlois, R. Henderson, Development of a Multilevel Multidisciplinary-Optimization Capability for an Industrial Environment, AIAA Journal 51 (10) (2013) 2335-2352. doi:10.2514/1. J052180.

URL https://doi.org/10.2514/1.J052180

[2] A. Rizzi, Modeling and simulating aircraft stability and controlThe SimSAC project, Progress in Aerospace Sciences 47 (8) (2011) 573-588. doi:10.1016/j.paerosci.2011.08.004. URL http://www.sciencedirect.com/science/article/pii/S0376042111000704

[3] C. P. van Dam, The aerodynamic design of multi-element high-lift systems for transport airplanes, Progress in Aerospace Sciences 38 (2) (2002) 101-144. doi:10.1016/S0376-0421(02)00002-7. URL http://www.sciencedirect.com/science/article/pii/S0376042102000027

[4] S. Gallay, E. Laurendeau, Nonlinear Generalized Lifting-Line Coupling Algorithms for Pre/Poststall Flows, AIAA Journal 53 (7) (2015) 1784-1792. doi:10.2514/1. J053530. URL https://doi.org/10.2514/1.J053530

[5] S. Gallay, E. Laurendeau, Preliminary-Design Aerodynamic Model for Complex Configurations Using Lifting-Line Coupling Algorithm, Journal of Aircraft 53 (4) (2016) 1145-1159. doi:10.2514/1. C033460. URL https://doi.org/10.2514/1.C033460

[6] S. Gallay, Algorithmes de couplage RANS et ecoulement potentiel, phd, Ecole Polytechnique de Montreal (Feb. 2016). URL https://publications.polymtl.ca/2061/

[7] R. Mukherjee, A. Gopalarathnam, S. W. Kim, An Iterative Decambering Approach for Post-Stall Prediction of Wing Characteristics using known Section Data, in: 41nd AIAA Aerospace Sciences 
Meeting and Exhibit, American Institute of Aeronautics and Astronautics, Reno, Nevada, 2003. URL http://archive.org/details/nasa_techdoc_20040085758

[8] J. N. Dias, Nonlinear Lifting-Line Algorithm for Unsteady and Post-stall Conditions, in: 34th AIAA Applied Aerodynamics Conference, American Institute of Aeronautics and Astronautics, 2017. URL https://arc.aiaa.org/doi/abs/10.2514/6.2016-4164

[9] J. Mariens, A. Elham, M. J. L. v. Tooren, Quasi-Three-Dimensional Aerodynamic Solver for Multidisciplinary Design Optimization of Lifting Surfaces, Journal of Aircraft 51 (2) (2014) 547-558. doi:10.2514/1.C032261.

URL https://doi.org/10.2514/1.C032261

[10] R. Hindman, N. Crist, B. Williams, X. Chi, Y. Choo, T. Shih, Q3d-Wing: Validation of a Modern Lifting-Line Method for Application to Clean and Iced Wings, in: 44th AIAA Aerospace Sciences Meeting and Exhibit, American Institute of Aeronautics and Astronautics, Reno, Nevada, 2006. doi : DOI : $10.2514 / 6.2006-1268$.

URL https://arc.aiaa.org/doi/abs/10.2514/6.2006-1268

[11] S. Gallay, S. Ghasemi, E. Laurendeau, Sweep effects on non-linear Lifting Line Theory near Stall, in: 52nd Aerospace Sciences Meeting, American Institute of Aeronautics and Astronautics, 2017. URL https://arc.aiaa.org/doi/abs/10.2514/6.2014-1105

[12] N. Hansen, A. Ostermeier, Completely derandomized self-adaptation in evolution strategies, Evolutionary Computation 9 (2) (2001) 159-195. doi:10.1162/106365601750190398.

[13] J. Katz, A. Plotkin, Low-Speed Aerodynamics, 2nd Edition, Cambridge University Press, Cambridge, 2001.

[14] E. Pistolesi, Considerations on the Mutual Interference of Aerofoil Systems.

[15] A. Pigeon, A. Levesque, E. Laurendeau, Two-dimensional navier-stokes flow solver developments at Ecole Polytechnique de Montreal, in: 22nd Annual Conference of the CFD Society of Canada, CFD Society of Canada, 2014.

[16] S. Bourgault-Ct, S. Ghasemi, A. Mosahebi, . Laurendeau, Extension of a Two-Dimensional NavierStokes Solver for Infinite Swept Flow, AIAA Journal 55 (2) (2017) 662-667. doi:10.2514/1. J055139. URL https://doi.org/10.2514/1.J055139

[17] D. Kchemann, A Simple Method for Calculating the Span and Chordwise Loading on Straight and Swept Wings of Any Given Aspect Ratio at Subsonic Speeds, Defense Technical Information Center, 1952.

[18] M. Parenteau, Aerodynamic optimization of aircraft wings using a coupled VLM-2.5d RANS approach, masters, cole Polytechnique de Montral (May 2017).

[19] M. Parenteau, K. Sermeus, E. Laurendeau, VLM Coupled with 2.5d RANS Sectional Data for High-Lift Design, in: 2018 AIAA Aerospace Sciences Meeting, American Institute of Aeronautics and Astronautics, Orlando, 2018, dOI: 10.2514/6.2018-1049.

[20] P. R. Spalart, Prediction of Lift Cells for Stalling Wings by Lifting-Line Theory, AIAA Journal 52 (8) (2014) 1817-1821. doi:10.2514/1.J053135. URL https://doi.org/10.2514/1.J053135

[21] W. O. Valarezo, V. D. Chin, Method for the prediction of wing maximum lift, Journal of Aircraft 31 (1) (1994) 103-109. doi:10.2514/3.46461.

URL https://doi.org/10.2514/3.46461

[22] D. A. Lovell, A Wind-Tunnel Investigation of the Effects of Flap Span and Deflection Angle, Wing Planform and a Body on the High-Lift Performance of a 28 degrees Swept Wing, Tech. rep., RAE (1977).

[23] D. Arnold, N. Hansen, A (1+1)-CMA-ES for Constrained Optimisation, in: GECCO, ACM Press, 2012, pp. 297-304. doi:10.1145/2330163.2330207.

URL https://hal.inria.fr/hal-00696268/document

[24] N. Hansen, S. Kern, Evaluating the CMA Evolution Strategy on Multimodal Test Functions, in: Parallel Problem Solving from Nature - PPSN VIII, Lecture Notes in Computer Science, Springer, Berlin, Heidelberg, 2004, pp. 282-291. doi:10.1007/978-3-540-30217-9_29. 
URL https://link.springer.com/chapter/10.1007/978-3-540-30217-9_29

[25] G. Collange, N. Delattre, N. Hansen, I. Quinquis, M. Schoenauer, Multidisciplinary Optimization in the Design of Future Space Launchers, in: P. Breitkopf, R. F. Coelho (Eds.), Multidisciplinary Design Optimization in Computational Mechanics, John Wiley \& Sons, Inc., 2013, pp. 459-468, dOI: 10.1002/9781118600153.ch12.

URL http://onlinelibrary.wiley.com/doi/10.1002/9781118600153.ch12/summary

[26] J. Mariens, Wing Shape Multidisciplinary Design Optimization, Ph.D. thesis, Delft University (2012). URL https://repository.tudelft.nl/islandora/object/uuid\%3A3b1c6432-cfbf-4fec-894b-9f6b870015f5

[27] E. Torenbeek, Synthesis of Subsonic Airplane Design: An introduction to the preliminary design of subsonic general aviation and transport aircraft, with emphasis on layout, aerodynamic design, propulsion and performance, 1982nd Edition, Springer, Delft : The Hague : Hingham, MA, 1982.

[28] A. Elham, G. La Rocca, M. J. L. van Tooren, An Advanced Quasi-Analytical Weight Estimation Method for Airplane Lifting Surfaces, in: 7th Annual Conference of Society of Allied Weight Engineers, Vol. 3571, Society of Allied Weight Engineers, Bavaria, Germany, 2012.

[29] J. Guay, Extension of the Overset Grid Preprocessor for Surface Conforming Meshes, masters, Ecole Polytechnique de Montreal (Apr. 2017).

URL https://publications.polymtl.ca/2525/

[30] M. Robitaille, A. Mosahebi, . Laurendeau, Design of adaptive transonic laminar airfoils using the -Ret transition model, Aerospace Science and Technology 46 (Supplement C) (2015) 60-71. doi: $10.1016 / j$.ast. 2015.06 .027 .

URL http://www.sciencedirect.com/science/article/pii/S1270963815002047

[31] FAA, 25-7c - Flight Test Guide For Certification Of Transport Category Airplanes (Oct. 2012). 